Z. Wojtkowiak

Nagoya Math. J.

Vol. 176 (2004), 113-158

\title{
ON $l$-ADIC ITERATED INTEGRALS, I ANALOG OF ZAGIER CONJECTURE
}

\author{
ZDZISŁAW WOJTKOWIAK
}

\begin{abstract}
We are studying some aspects of the action of Galois groups on the torsor of paths connecting two (possibly tangential) points on a projective line minus a finite number of points. We obtain objects which formally behave like classical iterated integrals and polylogarithms. We formulate an analog of Zagier conjecture for these $l$-adic analogs of iterated integrals and polylogarithms.
\end{abstract}

\section{$\S 0$. Introduction}

0.1. The classical complex iterated integrals appear in the study of mixed Hodge structures on fundamental groups and on torsors of paths (see [D], [BD] and [W3]). In this paper we shall study their $l$-adic analogs.

The notion of a tangential base point (see $[\mathrm{D}]$ ) is very important in this paper. We use a definition given in [N2].

Let $K$ be a number field and let $X$ be a projective line $\mathbf{P}_{K}^{1}$ minus a finite number of $K$-points. Let $z$ and $v$ be two $K$-points or tangential base points defined over $K$ of $X$. Let $\pi_{1}\left(X_{\bar{K}} ; v\right)$ be the $l$-completion of the étale fundamental group of $X_{\bar{K}}$ based at $v$. We denote by $\pi\left(X_{\bar{K}} ; z, v\right)$ the $\pi_{1}\left(X_{\bar{K}} ; v\right)$-torsor of (l-adic) paths from $v$ to $z$. The Galois group $G_{K}:=$ $\operatorname{Gal}(\bar{K} / K)$ acts on the set $\pi\left(X_{\bar{K}} ; z, v\right)$. To describe this action of $G_{K}$ we shall proceed in the following way.

Let us fix a path $p$ from $v$ to $z$. Then the map

$$
\pi\left(X_{\bar{K}} ; z, v\right) \ni q \longrightarrow p^{-1} q \in \pi_{1}\left(X_{\bar{K}} ; v\right)
$$

is a bijection. The action of $G_{K}$ on the torsor $\pi\left(X_{\bar{K}} ; z, v\right)$ transported to an action of $G_{K}$ on $\pi_{1}\left(X_{\bar{K}} ; v\right)$ by the map (0.1.1) is given by

$$
\pi_{1}\left(X_{\bar{K}} ; v\right) \ni S \longrightarrow \mathfrak{f}_{p}(\sigma) \cdot \sigma(S) \in \pi_{1}\left(X_{\bar{K}} ; v\right),
$$

Received January 7, 2003.

Revised March 10, 2003, July 23, 2003, October 14, 2003, March 10, 2004.

2000 Mathematics Subject Classification: 11G55, 11G99, $14 \mathrm{G} 32$. 
where $\sigma \in G_{K}$ and

$$
\mathfrak{f}_{p}(\sigma):=p^{-1} \cdot \sigma(p)
$$

The function $\mathfrak{f}_{p}: G_{K} \rightarrow \pi_{1}\left(X_{\bar{K}} ; v\right)$ has the following important property.

Proposition A. (see Section 1) The function $\mathfrak{f}_{p}: G_{K} \rightarrow \pi_{1}\left(X_{\bar{K}} ; v\right)$ is a cocycle, i.e.,

$$
\mathfrak{f}_{p}(\tau \cdot \sigma)=\mathfrak{f}_{p}(\tau) \cdot \tau\left(\mathfrak{f}_{p}(\sigma)\right)
$$

This (well known) result was the starting point of the paper (see also Theorem $\mathrm{A}$ and $\mathrm{B}$ in [I1]).

Let $X:=\mathbf{P}_{K}^{1} \backslash\left\{a_{1}, \ldots, a_{n}, \infty\right\}$. The fundamental group $\pi_{1}\left(X_{\bar{K}} ; v\right)$ is a pro- $l$ free group freely generated by $n$ generators, which we denote by $x_{1}, \ldots, x_{n}$ and which will be constructed below. The element $\mathfrak{f}_{p}(\sigma) \in$ $\pi_{1}\left(X_{\bar{K}} ; v\right)$, hence

$$
\begin{aligned}
\mathfrak{f}_{p}(\sigma) \equiv x_{1}^{\alpha_{1}(\sigma)} \cdot x_{2}^{\alpha_{2}(\sigma)} \cdots x_{n}^{\alpha_{n}(\sigma)} \cdot \prod_{i<j}\left(x_{i}, x_{j}\right)^{\beta_{i, j}(\sigma)} & \\
& \bmod \left(\left(\pi_{1}\left(X_{\bar{K}} ; v\right), \pi_{1}\left(X_{\bar{K}} ; v\right)\right), \pi_{1}\left(X_{\bar{K}} ; v\right)\right)
\end{aligned}
$$

for some $\alpha_{i}(\sigma)$ and $\beta_{i, j}(\sigma)$ in $\mathbf{Z}_{l}$. Let $G_{K}$ act on $\mathbf{Z}_{l}$ as a multiplication by the cyclotomic character $\chi: G_{K} \rightarrow \mathbf{Z}_{l}^{*}$. It follows from Proposition A that the exponents $\alpha_{i}: G_{K} \rightarrow \mathbf{Z}_{l}$ are cocycles (see Corollary 2.2.2). The obvious question is if the exponents $\beta_{i, j}: G_{K} \rightarrow \mathbf{Z}_{l}$ are also cocycles. This question and its generalization are studied in Sections 6 and 11.

The fundamental group $\pi_{1}\left(X_{\bar{K}} ; v\right)$ we embed into the algebra $\mathbf{Q}_{l}\left\{\left\{X_{1}\right.\right.$, $\left.\ldots, X_{n}\right\}$ \} of non-commutative formal power series in $n$ non-commuting variables $X_{1}, \ldots, X_{n}\left(n+1\right.$ is a number of points removed from $\left.\mathbf{P}_{K}^{1}\right)$ sending a loop around $a_{i}$ onto $e^{X_{i}}$ for $i=1, \ldots, n$. The actions of $G_{K}$ on the fundamental group $\pi_{1}\left(X_{\bar{K}} ; v\right)$ and on the torsor $\pi\left(X_{\bar{K}} ; z, v\right)$ we transport to linear actions of $G_{K}$ on $\mathbf{Q}_{l}\left\{\left\{X_{1}, \ldots, X_{n}\right\}\right\}$. Hence we get representations

$$
\varphi: G_{K} \longrightarrow \operatorname{Aut}\left(\mathbf{Q}_{l}\left\{\left\{X_{1}, \ldots, X_{n}\right\}\right\}\right)
$$

in a case of the action deduced from the action on $\pi_{1}\left(X_{\bar{K}} ; v\right)$ and

$$
\psi_{p}: G_{K} \longrightarrow \mathrm{GL}\left(\mathbf{Q}_{l}\left\{\left\{X_{1}, \ldots, X_{n}\right\}\right\}\right)
$$

in a case of the action deduced from the action on the torsor $\pi\left(X_{\bar{K}} ; z, v\right)$. 
If $\sigma \in G_{K\left(\mu_{l} \infty\right)}:=\operatorname{Gal}\left(\bar{K} / K\left(\mu_{l^{\infty}}\right)\right)$ then $\psi_{p}(\sigma)$ is a pro-unipotent automorphism of $\mathbf{Q}_{l}\left\{\left\{X_{1}, \ldots, X_{n}\right\}\right\}$. Hence $\log \psi_{p}(\sigma)$ is defined and we have the following result.

Proposition B. (see Section 5) Let $\sigma \in G_{K\left(\mu_{l} \infty\right)}$. Then we have

$$
\log \psi_{p}(\sigma)=L_{\left(\log \psi_{p}(\sigma)\right)(1)}+\log \varphi(\sigma),
$$

where for $w \in \mathbf{Q}_{l}\left\{\left\{X_{1}, \ldots, X_{n}\right\}\right\}, L_{w}$ is a left multiplication by $w$.

The operator $\log \varphi(\sigma)$ is a derivation of the $\mathbf{Q}_{l}$-algebra $\mathbf{Q}_{l}\left\{\left\{X_{1}, \ldots\right.\right.$, $\left.\left.X_{n}\right\}\right\}$. Let us fix a path $\gamma_{i}$ from $v$ to a tangential base point at $a_{i}$ for $i=1, \ldots, n$. The generator

$$
x_{i}:=\gamma_{i}^{-1} \cdot \text { small loop around } a_{i} \cdot \gamma_{i}
$$

we send to $e^{X_{i}}$ for $i=1, \ldots, n$. Then we show the following result.

Proposition C. (see Section 5) Let $\sigma \in G_{K\left(\mu_{l} \infty\right)}$. Then we have

$$
(\log \varphi(\sigma))\left(X_{i}\right)=\left[X_{i},\left(\log \psi_{\gamma_{i}}(\sigma)\right)(1)\right]
$$

for $i=1, \ldots, n$.

P. Deligne in [D] and Y. Ihara in [I1], [I2] have studied the Galois action on $\mathbf{P}^{1} \backslash\{0,1, \infty\}$. They got results related to our Proposition $\mathrm{C}$. Their results in the case of $\mathbf{P}^{1} \backslash\{0,1, \infty\}$ motivated our study of more general situations.

The power series $\left(\log \psi_{p}(\sigma)\right)(1)$ is a Lie element and its coefficients (with $\sigma \in G_{K\left(\mu_{l} \infty\right)}$ varing) in a Hall base we shall call $l$-adic iterated integrals (see Definition 5.3.0). These $l$-adic iterated integrals are functions from $G_{K\left(\mu_{l} \infty\right)}$ to $\mathbf{Q}_{l}$. They depend on points $v$ and $z$ and also on a choice of a path $p$ from $v$ to $z$ (compare with the classical integral $\int_{v}^{z} \frac{d z}{z}$ which depends on $v$ and $z$ and on a choice of a path $p$ from $v$ to $z$ ). They have all formal properties of iterated integrals on $X(\mathbf{C})$. In [W1] we studied functional equations of iterated integrals. The $l$-adic iterated integrals have the same functional equations as classical complex iterated integrals on $X(\mathbf{C})$ (see Section 10). We have an analog of Zagier conjecture for $l$-adic iterated integrals as in [W3] (see Section 7).

We would like to thank H. Nakamura for discussions of some topics considered in the paper and and for encouragements. The results of this 
paper were presented in unofficial talk in Berkeley and in Oberwolfach in autumn 1999, in Luminy in spring 2000 and in summer 2001, in Acquafreda di Maretea in 2001 and in Cambridge in autumn 2002.

The present paper is a rewritten version of the first six sections of [W4].

\section{$\S 1$. Torsors of paths}

1.0. Let $X$ be a smooth algebraic variety defined over a number field $K$. We denote by $\hat{X}(K)$ the union of $K$-points of $X$ and tangential base points of $X$ defined over $K$.

Let us fix a prime number $l$. Let $z, v \in \hat{X}(K)$. Let $\pi_{1}\left(X_{\bar{K}} ; v\right)$ be the $l$-completion, i.e., the maximal pro- $l$ quotient of the étale fundamental group of $X_{\bar{K}}$ with a base point at $v$. We denote by $\pi\left(X_{\bar{K}} ; z, v\right)$ the profinite set of homotopy classes of (l-adic) paths from $v$ to $z$. The set $\pi\left(X_{\bar{K}} ; z, v\right)$ is a $\pi_{1}\left(X_{\bar{K}} ; v\right)$-torsor. We set $G_{K}:=\operatorname{Gal}(\bar{K} / K)$. The group $G_{K}$ acts on $\pi_{1}\left(X_{\bar{K}} ; v\right)$ and on $\pi\left(X_{\bar{K}} ; z, v\right)$ and the action of $G_{K}$ is compatible with the action of $\pi_{1}\left(X_{\bar{K}} ; v\right)$ on $\pi\left(X_{\bar{K}} ; z, v\right)$, i.e., $\sigma(p \cdot S)=\sigma(p) \cdot \sigma(S)$ for $p \in$ $\pi\left(X_{\bar{K}} ; z, v\right), S \in \pi_{1}\left(X_{\bar{K}} ; v\right)$ and $\sigma \in G_{K}$.

In this section we shall study elementary properties of the action of the Galois group $G_{K}$ on the torsor of paths $\pi\left(X_{\bar{K}} ; z, v\right)$. The set $\pi\left(X_{\bar{K}} ; z, v\right)$ is difficult to handle. We fix a path from $v$ to $z$ and using this path we identify the set $\pi\left(X_{\bar{K}} ; z, v\right)$ with the fundamental group $\pi_{1}\left(X_{\bar{K}} ; v\right)$. The group $\pi_{1}\left(X_{\bar{K}} ; v\right)$ is more familiar and we describe the action of $G_{K}$ on $\pi\left(X_{\bar{K}} ; z, v\right)$ in terms of the action of $G_{K}$ on $\pi_{1}\left(X_{\bar{K}} ; v\right)$.

Let us fix a path $p \in \pi\left(X_{\bar{K}} ; z, v\right)$. Then

$$
t_{p}: \pi\left(X_{\bar{K}} ; z, v\right) \longrightarrow \pi_{1}\left(X_{\bar{K}} ; v\right)
$$

given by $t_{p}(q):=p^{-1} \cdot q$ is a bijection. The map $t_{p}$ is not $G_{K}$-equivariant. However using this map we shall transport the action of $G_{K}$ on $\pi\left(X_{\bar{K}} ; z, v\right)$ into the action of $G_{K}$ on $\pi_{1}\left(X_{\bar{K}} ; v\right)$, which is a more familiar object.

Let $\sigma \in G_{K}$. We set

$$
\sigma_{p}:=t_{p} \circ \sigma \circ t_{p}^{-1}
$$

where $\sigma: \pi\left(X_{\bar{K}} ; z, v\right) \rightarrow \pi\left(X_{\bar{K}} ; z, v\right)$ is the map induced by $\sigma$.

Definition 1.0.1. We define a function $\mathfrak{f}_{p}: G_{K} \rightarrow \pi_{1}\left(X_{\bar{K}} ; v\right)$ setting

$$
\mathfrak{f}_{p}(\sigma):=p^{-1} \cdot \sigma(p) \in \pi_{1}\left(X_{\bar{K}} ; v\right)
$$

for any $\sigma \in G_{K}$. 
LEMMA 1.0.2. The action of $G_{K}$ on $\pi_{1}\left(X_{\bar{K}} ; v\right)$ transported by the isomorphism $t_{p}$ from the action of $G_{K}$ on $\pi\left(X_{\bar{K}} ; z, v\right)$ is given by

$$
\sigma_{p}(S)=\mathfrak{f}_{p}(\sigma) \cdot \sigma(S)
$$

where $S \in \pi_{1}\left(X_{\bar{K}} ; v\right)$ and $\sigma \in G_{K}$.

Proof. We have $\sigma_{p}(S)=t_{p} \circ \sigma \circ t_{p}^{-1}(S)=t_{p}(\sigma(p \cdot S))=p^{-1} \cdot \sigma(p) \cdot \sigma(S)=$ $\mathfrak{f}_{p}(\sigma) \cdot \sigma(S)$.

This action of $G_{K}$ on $\pi_{1}\left(X_{\bar{K}} ; v\right)$ transported by the isomorphism $t_{p}$ depends on a choice of a path $p$ from $v$ to $z$. Let $q \in \pi\left(X_{\bar{K}} ; z, v\right)$ be another path from $v$ to $z$. One easily verifies that

$$
\mathfrak{f}_{p}(\sigma)=\left(q^{-1} p\right)^{-1} \cdot \mathfrak{f}_{q}(\sigma) \cdot \sigma\left(q^{-1} p\right)
$$

and

$$
t_{p}(r)=t_{q}\left(\left(q p^{-1}\right) \cdot r\right)=\left(p^{-1} q\right) \cdot t_{q}(r)
$$

for any $r$ in $\pi\left(X_{\bar{K}} ; z, v\right)$.

The relation between actions of $\sigma_{p}$ and $\sigma_{q}$ is described in the next lemma.

LEMmA 1.0.5. For any $\sigma \in G_{K}$ and $S \in \pi_{1}\left(X_{\bar{K}} ; v\right)$ we have

$$
\sigma_{p}(S)=\left(q^{-1} p\right)^{-1} \cdot \sigma_{q}\left(\left(q^{-1} p\right) \cdot S\right)
$$

Proof. The lemma follows from Lemma 1.0.2 and from (1.0.3).

We finish this section describing some elementary properties of the element $\mathfrak{f}_{p}(\sigma)$.

LEMMA 1.0.6. Let $p$ be a path from $v$ to $z$ and let $q$ be a path from $w$ to $v$. Then we have

$$
\mathfrak{f}_{p q}(\sigma)=q^{-1} \cdot \mathfrak{f}_{p}(\sigma) \cdot q \cdot \mathfrak{f}_{q}(\sigma) \quad \text { and } \quad \mathfrak{f}_{p^{-1}}(\sigma)=p \cdot\left(\mathfrak{f}_{p}(\sigma)\right)^{-1} \cdot p^{-1}
$$

for any $\sigma \in G_{K}$.

Proof. An easy verification we left to the reader. 
Proposition 1.0.7. The function $\mathfrak{f}_{p}: G_{K} \rightarrow \pi_{1}\left(X_{\bar{K}} ; v\right)$ is a cocycle, i.e., for any $\tau$ and $\sigma$ in $G_{K}$ we have

$$
\mathfrak{f}_{p}(\tau \cdot \sigma)=\mathfrak{f}_{p}(\tau) \cdot \tau\left(\mathfrak{f}_{p}(\sigma)\right)
$$

Proof. We have $\mathfrak{f}_{p}(\tau \cdot \sigma)=p^{-1} \cdot \tau(\sigma(p))=p^{-1} \cdot \tau(p) \cdot \tau\left(p^{-1}\right) \cdot \tau(\sigma(p))=$ $\mathfrak{f}_{p}(\tau) \cdot \tau\left(\mathfrak{f}_{p}(\sigma)\right)$.

Corollary 1.0.8. We have

$$
\mathfrak{f}_{p}\left(\tau^{-1}\right)=\tau^{-1}\left(\mathfrak{f}_{p}(\tau)^{-1}\right)
$$

Remark. Let $p$ be a path from $\overrightarrow{01}$ to $\overrightarrow{10}$ on $\mathbf{P}^{1}(\mathbf{C}) \backslash\{0,1, \infty\}$. The element $\mathfrak{f}_{p}(\sigma)$ was used by Ihara in [I2]. Its Hodge-De Rham incarnation appears in $[\mathrm{D}]$ and $[\mathrm{Dr}]$.

\section{$\S 2$. Geometric generators of $\pi_{1}(X(\mathbf{C}) ; v)$}

2.0. Let $X=\mathbf{P}_{\mathbf{C}}^{1} \backslash\left\{a_{1}, \ldots, a_{n+1}\right\}$ and let $v \in \hat{X}(\mathbf{C})$. We shall construct a canonical family of generators of $\pi_{1}(X(\mathbf{C}) ; v)$. The Galois action on fundamental groups will be described in terms of these generators.

Let us choose a tangential base point $v_{i}$ (a tangent vector) at $a_{i}$ for $i=1,2, \ldots, n+1$.

2.0.1. Let us assume that $v \in X(\mathbf{C})$. Let $\Gamma=\left\{\gamma_{k}\right\}_{k=1, \ldots, n+1}$ be a family of smooth paths from $v$ to each $v_{k}$ such that any two paths do not intersect, no path self-intersects and for each $k, \gamma_{k}([0,1[) \subset X(\mathbf{C})$. The indices are choosen in such a way that when we make a small circle around $v$ in the opposite clockwise direction starting from $\gamma_{1}$, then we meet successively $\gamma_{2}, \gamma_{3}, \ldots, \gamma_{n+1}$. The element $S_{k} \in \pi_{1}(X(\mathbf{C}) ; v)$ is defined in the following way: we move along $\gamma_{k}$, near $a_{k}$ we make a small circle around $a_{k}$ in the opposite clockwise direction and we return along $\gamma_{k}$ to $v$ (see Picture 1).

2.0.2. Without loss of generality we can assume that $v$ is a tangential base point at $a_{1}$. Let $v^{\prime} \in X(\mathbf{C})$ be near $a_{1}$ in the direction $v$. Let $\Gamma=$ $\left\{\gamma_{k}^{\prime}\right\}_{k=2, \ldots, n+1}$ be a family of smooth paths from $v^{\prime}$ to each $v_{k}$ satisfying the conditions from 2.0.1. Let $S_{k}^{\prime}$ be defined by the path $\gamma_{k}^{\prime}$. Let $\gamma$ be a path $[0,1] \ni t \rightarrow a_{1}+t\left(v^{\prime}-a_{1}\right) \in X(\mathbf{C})$. We set $\gamma_{k}:=\gamma_{k}^{\prime} \cdot \gamma$ and $S_{k}:=\gamma^{-1} \cdot S_{k}^{\prime} \cdot \gamma$ for $k=2, \ldots, n+1$. $S_{1}$ is a small circle around $a_{1}$ starting from $v$ in the opposite clockwise direction (see Picture 2). 


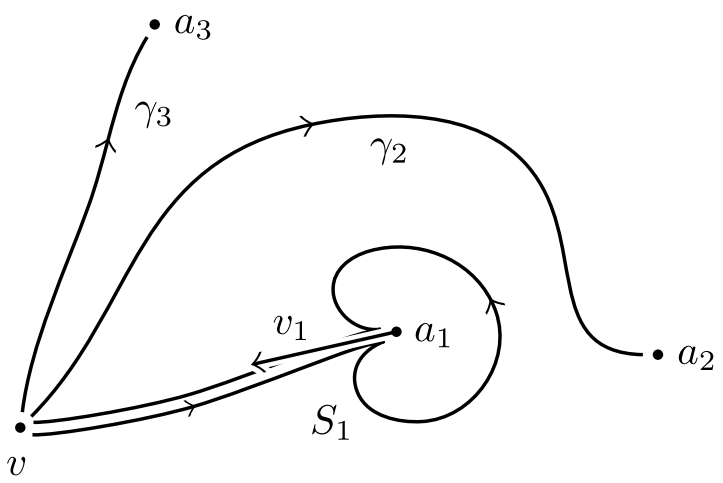

Picture 1

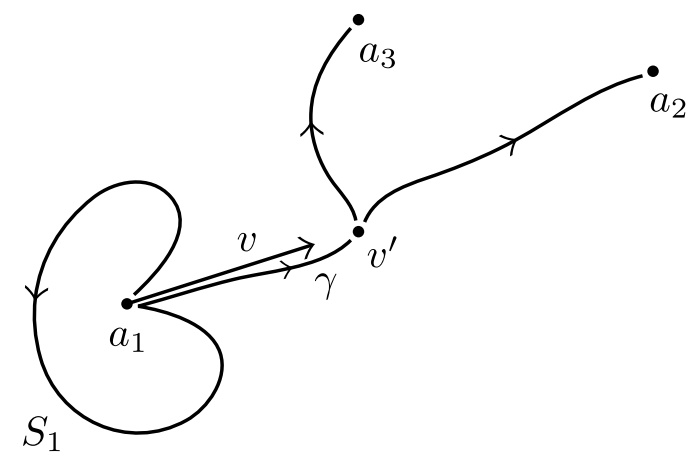

Picture 2

LEMMA 2.0.3. The elements $S_{1}, \ldots, S_{n+1}$ generate $\pi_{1}(X(\mathbf{C}) ; v)$ and satisfy the only relation

$$
S_{n+1} \cdots S_{1}=1
$$

Definition 2.0.4. The ordered sequence $\left(S_{1}, \ldots, S_{n+1}\right)$ we shall call a sequence of geometric generators of $\pi_{1}(X(\mathbf{C}) ; v)$ associated to a family of paths $\Gamma$.

2.1. Let $F_{n+1}=F_{n+1}\left(x_{1}, \ldots, x_{n+1}\right)$ be a free group on $n+1$ elements $\left(x_{1}, \ldots, x_{n+1}\right)$. Let $\mathcal{B}_{n+1}\left(x_{1}, \ldots, x_{n+1}\right)$ be a subgroup of $\operatorname{Aut}\left(F_{n+1}\right)$ consisting of automorphisms $f$ such that $f\left(x_{i}\right)=t_{i} \cdot x_{\mu(i)} \cdot t_{i}^{-1}(i=1, \ldots, n+1)$ and $f\left(x_{n+1}\right) \cdots f\left(x_{1}\right)=x_{n+1} \cdots x_{1}$, where $t_{i} \in F_{n+1}$ and $\mu \in S_{n+1}$ is a permutation.

Let us set $F_{n+1}^{*}:=F_{n+1} /\left\langle x_{n+1} \cdots x_{1}\right\rangle$. The group $\mathcal{B}_{n+1}\left(x_{1}, \ldots, x_{n+1}\right)$ acts as an automorphism group on $F_{n+1}^{*}$. This automorphism group we 
denote by $\mathcal{B}_{n+1}^{*}\left(x_{1}, \ldots, x_{n+1}\right)$. Let

$$
\mathcal{B}_{n+1}^{(1) *}\left(x_{1}, \ldots, x_{n+1}\right):=\operatorname{ker}\left(\pi: \mathcal{B}_{n+1}^{*}\left(x_{1}, \ldots, x_{n+1}\right) \rightarrow \Sigma_{n+1}\right),
$$

where $\pi$ is the obvious projection.

The next lemma is well known.

LEMMA 2.1.1. (see [W2]) Let $\left(S_{1}, \ldots, S_{n+1}\right)$ be a sequence of geometric generators of $\pi_{1}(X(\mathbf{C}) ; v)$. Then any other sequence of geometric generators of $\pi_{1}(X(\mathbf{C}) ; v)$ is of the form $\left(f\left(S_{1}\right), \ldots, f\left(S_{n+1}\right)\right)$, where $f \in$ $\mathcal{B}_{n+1}^{*}\left(S_{1}, \ldots, S_{n+1}\right)$.

Definition 2.1.2. Let $s=\left(S_{1}, \ldots, S_{n+1}\right)$ and $s^{\prime}=\left(S_{1}^{\prime}, \ldots, S_{n+1}^{\prime}\right)$ be two sequences of geometric generators of $\pi_{1}(X(\mathbf{C}) ; v)$. We say that $s$ and $s^{\prime}$ are in the same permutation class if there is $f \in \mathcal{B}_{n+1}^{(1) *}\left(S_{1}, \ldots, S_{n+1}\right)$ such that $f\left(S_{i}\right)=S_{i}^{\prime}$ for each $i$.

2.2. Let $K$ be a number field. Let $a_{1}, \ldots, a_{n+1}$ be $K$-points of the projective line $\mathbf{P}_{K}^{1}$. Let $X=\mathbf{P}_{K}^{1} \backslash\left\{a_{1}, \ldots, a_{n+1}\right\}$ and let $v \in \hat{X}(K)$. Let us choose a tangential base point $v_{k} \in \hat{X}(K)$ at $a_{k}$ for $k=1, \ldots, n+1$. Let us fix an embedding $K \subset \mathbf{C}$. Let $\Gamma=\left\{\gamma_{k}\right\}_{k=1, \ldots, n+1}$ be a family of paths on $X(\mathbf{C})$ from $v$ to each $v_{k}$ and let $S_{1}, \ldots, S_{n+1}$ be a family of geometric generators of $\pi_{1}(X(\mathbf{C}) ; v)$ associated to $\Gamma$.

The geometric generators of $\pi_{1}(X(\mathbf{C}) ; v)$ can be interpreted as elements of $\pi_{1}\left(X_{\bar{K}} ; v\right)$. The path $\gamma_{k}$ from $v$ to $v_{k}$ can be interpreted as an $l$-adic path, i.e., a natural transformation of fiber functors over $v$ and over $v_{k}$ from étale coverings of $X_{\bar{K}}$ to sets. A small circle around $a_{k}$ based at $v_{k}$ is defined in the proof of Proposition 2.2.1. However it would be very interesting to construct "geometric generators" of $\pi_{1}\left(X_{\bar{K}} ; v\right)$ in purely algebraic way.

Below we shall describe the action of $G_{K}$ on $\pi_{1}\left(X_{\bar{K}} ; v\right)$ in terms of these generators. The result seems to be well known (see [I1, pages 51 and 52] and [AI, page 128]). We give however a sketch of a proof because of the importance of this result in our studies.

Let $\chi: G_{K} \rightarrow \mathbf{Z}_{l}^{*}$ be the cyclotomic character.

Proposition 2.2.1. Let $\sigma \in G_{K}$. Then

$$
\sigma\left(S_{k}\right)=\left(\mathfrak{f}_{\gamma_{k}}(\sigma)\right)^{-1} \cdot S_{k}^{\chi(\sigma)} \cdot \mathfrak{f}_{\gamma_{k}}(\sigma)
$$

for $k=1, \ldots, n+1$. 
Proof. Without loss of generality we can assume that $a_{k}=0, a_{n+1}=$ $\infty$ and $v_{k}=\overrightarrow{01}$. Consider the following Galois equivariant map

$$
\pi_{1}\left(\operatorname{Spec} \bar{K}[[z]]\left[\frac{1}{z}\right], \overrightarrow{01}\right) \longrightarrow \pi_{1}\left(X_{\bar{K}}, v_{k}\right),
$$

where $\bar{K}[[z]]\left[\frac{1}{z}\right]$ is the algebra of formal Laurent power series. The fundamental group $\pi_{1}\left(\operatorname{Spec} \bar{K}[[z]]\left[\frac{1}{z}\right], \overrightarrow{01}\right)$ is isomorphic to $\mathbf{Z}_{l}$. The group $G_{K}$ acts on $\pi_{1}\left(\operatorname{Spec} \bar{K}[[z]]\left[\frac{1}{z}\right], \overrightarrow{01}\right)$ by the cyclotomic character $\chi: G_{K} \rightarrow \mathbf{Z}_{l}^{*}$. (See [I1] and [N1, p. 94].)

Let us fix an embedding of $\bar{K}$ into C. We recall that the elements of $\pi_{1}\left(\operatorname{Spec} \bar{K}[[z]]\left[\frac{1}{z}\right], \overrightarrow{01}\right)$ act on Puiseux elements $z^{1 / l^{n}}$ by analytic continuation. We define a canonical generator $T$ of $\pi_{1}\left(\operatorname{Spec} \bar{K}[[z]]\left[\frac{1}{z}\right], \overrightarrow{01}\right)$ requiring that $T\left(z^{1 / l^{n}}\right)=e^{2 \pi i / l^{n}} \cdot z^{1 / l^{n}}$ (see Picture 3$)$.

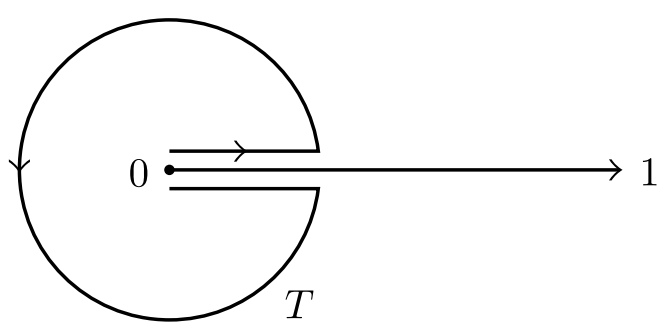

Picture 3

We denote by $T_{k}$ the image of $T$ in $\pi_{1}\left(X_{\bar{K}}, v_{k}\right)$. Clearly we have $\sigma\left(T_{k}\right)=$ $T_{k}^{\chi(\sigma)}$. Observe that $S_{k}=\gamma_{k}^{-1} \cdot T_{k} \cdot \gamma_{k}$. Hence we get $\sigma\left(S_{k}\right)=\sigma\left(\gamma_{k}^{-1}\right) \cdot T_{k}^{\chi(\sigma)}$. $\sigma\left(\gamma_{k}\right)=\sigma\left(\gamma_{k}^{-1}\right) \cdot \gamma_{k} \cdot\left(\gamma_{k}^{-1} \cdot T_{k}^{\chi(\sigma)} \cdot \gamma_{k}\right) \cdot\left(\gamma_{k}^{-1}\right) \cdot \sigma\left(\gamma_{k}\right)=\left(\mathfrak{f}_{\gamma_{k}}(\sigma)\right)^{-1} \cdot S_{k}^{\chi(\sigma)} \cdot \mathfrak{f}_{\gamma_{k}}(\sigma)$.

Let $z \in \hat{X}(K)$ and let $p$ be a path from $v$ to $z$. Let us define functions $\alpha_{i}: G_{K} \rightarrow \mathbf{Z}_{l}$ for $i=1,2, \ldots, n$ by the following congruence

$$
\mathfrak{f}_{p}(\sigma) \equiv \prod_{i=1}^{n} S_{i}^{\alpha_{i}(\sigma)} \bmod \left(\pi_{1}\left(X_{\bar{K}} ; v\right), \pi_{1}\left(X_{\bar{K}} ; v\right)\right)
$$

Let $G_{K}$ act on $\mathbf{Z}_{l}$ as a multiplication by the cyclotomic character $\chi: G_{K} \rightarrow$ $\mathbf{Z}_{l}^{*}$.

Corollary 2.2.2. The functions $\alpha_{i}: G_{K} \rightarrow \mathbf{Z}_{l}$ for $i=1,2, \ldots, n$ are cocycles.

Proof. The corollary follows from Propositions 1.0.7 and 2.2.1. 


\section{§3. Filtrations of $G_{K}$ associated with the lower central series of $\pi_{1}$}

3.0. In this section we shall study various filtrations of the group $G_{K}$ obtained from the action of $G_{K}$ on fundamental groups and on torsors of paths. The filtrations obtained from the action on fundamental groups were already studied by Ihara (see [I1]), Nakamura and Tsunogai (see [NT]) and others.

These filtrations are associated to the lower central series filtrations. Hence we recall here the definition of the lower central series of a group.

Let $\pi$ be a group. The subgroups $\Gamma^{n} \pi$ of the lower central series are defined recursively by

$$
\Gamma^{1} \pi:=\pi, \quad \Gamma^{n+1} \pi:=\left(\Gamma^{n} \pi, \pi\right), \quad n=1,2, \ldots
$$

(see [MKS, Section 5.3]).

Let $X=\mathbf{P}_{K}^{1} \backslash\left\{a_{1}, \ldots, a_{n+1}\right\}$ and let $z, v \in \hat{X}(K)$. Fix an embedding of $\bar{K}$ into C. Let $x=\left(x_{1}, \ldots, x_{n+1}\right)$ be a sequence of geometric generators of $\pi_{1}(X(\mathbf{C}) ; v)$ associated with a family of paths $\Gamma=\left\{\gamma_{i}\right\}_{i=1, \ldots, n+1}$. The action of $G_{K}$ on $\pi_{1}\left(X_{\bar{K}} ; v\right)$ preserves $\Gamma^{i+1} \pi_{1}\left(X_{\bar{K}} ; v\right)$, hence $G_{K}$ acts also on the quotient group $\pi_{1}\left(X_{\bar{K}} ; v\right) / \Gamma^{i+1} \pi_{1}\left(X_{\bar{K}} ; v\right)$.

We set

$$
G_{i}=G_{i}(X, v):=\operatorname{ker}\left(G_{K} \rightarrow \operatorname{Aut}\left(\pi_{1}\left(X_{\bar{K}} ; v\right) / \Gamma^{i+1} \pi_{1}\left(X_{\bar{K}} ; v\right)\right)\right)
$$

Observe that $G_{1}=\operatorname{Gal}\left(\bar{K} / K\left(\mu_{l^{\infty}}\right)\right)$. The quotient group $G_{i} / G_{i+1}$ is isomorphic to a finite direct sum of several copies of $\mathbf{Z}_{l}$ (see $[\mathrm{NT}$, Theorem (5.11)]). This implies that $G_{k} / G_{i}$ are $l$-adic Lie groups.

The group $G_{K} / G_{1} \subset \mathbf{Z}_{l}^{*}$ acts on $G_{i} / G_{i+1}$ and the $G_{K} / G_{1}$-module $G_{i} / G_{i+1}$ is isomorphic to $\mathbf{Z}_{l}(i)^{n_{i}}$ (see [I1] in the special case, when $X=$ $\left.\mathbf{P}_{\mathbf{Q}}^{1} \backslash\{0,1, \infty\}\right)$. Below we shall show that this result is a corollary of a more general statement.

Let us set $G_{\infty}=G_{\infty}(X, v):=\bigcap_{i=1}^{\infty} G_{i}(X, v)$. Then $G_{1} / G_{\infty}=$ $\lim _{i} G_{1} / G_{i}$ is a pro $l$-adic Lie group.

We say that two paths $p, q \in \pi\left(X_{\bar{K}} ; z, v\right)$ are $\Gamma^{i}$-equivalent if $p^{-1}$. $q \in \Gamma^{i} \pi_{1}\left(X_{\bar{K}} ; v\right)$. The set of $\Gamma^{i}$-equivalence classes, which we denote by $\pi\left(X_{\bar{K}} ; z, v\right) / \Gamma^{i}$, is a $\pi_{1}\left(X_{\bar{K}} ; v\right) / \Gamma^{i} \pi_{1}\left(X_{\bar{K}} ; v\right)$-torsor. The action of $G_{K}$ on $\pi\left(X_{\bar{K}} ; z, v\right)$ induces an action of $G_{K}$ on $\pi\left(X_{\bar{K}} ; z, v\right) / \Gamma^{i}$ compatible with the structure of the $\pi_{1}\left(X_{\bar{K}} ; v\right) / \Gamma^{i} \pi_{1}\left(X_{\bar{K}} ; v\right)$-torsor. 
We introduce a subgroup $H_{i}=H_{i}(X ; z, v)$ of $G_{i}$ by

$$
H_{i}=H_{i}(X ; z, v):=\operatorname{ker}\left(G_{i}(X, v) \rightarrow \operatorname{Aut}_{S e t}\left(\pi\left(X_{\bar{K}} ; z, v\right) / \Gamma^{i}\right)\right) .
$$

Proposition 3.0.1. The conjugation on $H_{j}$ by elements of $G_{K}$ induces an action of $G_{K} / G_{1} \subset \mathbf{Z}_{l}^{*}$ on the quotient group $H_{j} / H_{j+1}$. Moreover $H_{j} / H_{j+1}$ is isomorphic to a finite direct sum $\mathbf{Z}_{l}(j)^{m_{j}}$ as a $G_{K} / G_{1}$-module.

Proof. Let us fix a path $p$ from $v$ to $z$. The map $t_{p}: \pi\left(X_{\bar{K}} ; z, v\right) \rightarrow$ $\pi_{1}\left(X_{\bar{K}} ; v\right)$ is $G_{K}$-equivariant, if $\sigma \in G_{K}$ acts by $\sigma_{p}$ on $\pi_{1}\left(X_{\bar{K}} ; v\right)$. The map $t_{p}$ induces a $G_{K}$-equivariant map

$$
\pi\left(X_{\bar{K}} ; z, v\right) / \Gamma^{j} \pi\left(X_{\bar{K}} ; z, v\right) \rightarrow \pi_{1}\left(X_{\bar{K}} ; v\right) / \Gamma^{j} \pi_{1}\left(X_{\bar{K}} ; v\right) .
$$

Hence we get that

$$
H_{j}=\operatorname{ker}\left(G_{j} \rightarrow \operatorname{Aut}_{S e t}\left(\pi_{1}\left(X_{\bar{K}} ; v\right) / \Gamma^{j} \pi_{1}\left(X_{\bar{K}} ; v\right)\right)\right) .
$$

Let $\sigma \in H_{j}$. Proposition 2.2.1 implies that $\sigma\left(x_{k}\right)=\left(\mathfrak{f}_{\gamma_{k}}(\sigma)\right)^{-1} \cdot x_{k} \cdot \mathfrak{f}_{\gamma_{k}}(\sigma)$ for $k=1, \ldots, n, n+1$. Observe that $\mathfrak{f}_{\gamma_{k}}(\sigma) \in \Gamma^{j} \pi_{1}\left(X_{\bar{K}} ; v\right)$ for $k=1, \ldots, n, n+1$ and $\mathfrak{f}_{p}(\sigma) \in \Gamma^{j} \pi_{1}\left(X_{\bar{K}} ; v\right)$. The sequence

$$
\left(\mathfrak{f}_{p}(\sigma), \mathfrak{f}_{\gamma_{1}}(\sigma), \ldots, \mathfrak{f}_{\gamma_{n}}(\sigma)\right) \in \Gamma^{j} \pi_{1}\left(X_{\bar{K}} ; v\right) \times\left(\Gamma^{j} \pi_{1}\left(X_{\bar{K}} ; v\right)\right)^{n}
$$

determines the map $\sigma_{p}$. This implies that the quotient group $H_{j} / H_{j+1}$ is isomorphic to a closed subgroup of

$$
\Gamma^{j} \pi_{1}\left(X_{\bar{K}} ; v\right) / \Gamma^{j+1} \pi_{1}\left(X_{\bar{K}} ; v\right) \times\left(\Gamma^{j} \pi_{1}\left(X_{\bar{K}} ; v\right) / \Gamma^{j+1} \pi_{1}\left(X_{\bar{K}} ; v\right)\right)^{n} .
$$

Therefore the quotient group $H_{j} / H_{j+1}$ is isomorphic to a finite direct sum $\mathbf{Z}_{l}^{m_{j}}$.

Let $\tau \in G_{K}$ and $\sigma \in H_{j}$. We shall show that $\tau \cdot \sigma \cdot \tau^{-1}=\chi(\tau)^{j} \cdot \sigma$ in $H_{j} / H_{j+1}$. It follows from Proposition 1.0.7 and Corollary 1.0.8 that $\mathfrak{f}_{p}\left(\tau \cdot \sigma \cdot \tau^{-1}\right)=\mathfrak{f}_{p}(\tau) \cdot \tau\left(\mathfrak{f}_{p}(\sigma)\right) \cdot\left(\tau \cdot \sigma \cdot \tau^{-1}\right)\left(\mathfrak{f}_{p}(\tau)^{-1}\right)$. Observe that $\mathfrak{f}_{p}(\tau)$. $\tau\left(\mathfrak{f}_{p}(\sigma)\right) \cdot\left(\tau \cdot \sigma \cdot \tau^{-1}\right)\left(\mathfrak{f}_{p}(\tau)^{-1}\right)=\tau\left(\mathfrak{f}_{p}(\sigma)\right) \bmod \Gamma^{j+1} \pi_{1}\left(X_{\bar{K}} ; v\right)$ and $\tau\left(\mathfrak{f}_{p}(\sigma)\right)=$ $\chi(\tau)^{j} \cdot \mathfrak{f}_{p}(\sigma) \bmod \Gamma^{j+1} \pi_{1}\left(X_{\bar{K}} ; v\right)$. This implies the proposition because we have also

$$
\mathfrak{f}_{\gamma_{i}}\left(\tau \cdot \sigma \cdot \tau^{-1}\right)=\chi(\tau)^{j} \cdot \mathfrak{f}_{\gamma_{i}}(\sigma) \bmod \Gamma^{j+1} \pi_{1}\left(X_{\bar{K}} ; v\right)
$$

for $i=1, \ldots, n, n+1$. 
Corollary 3.0.2. The conjugation on $G_{j}$ by elements of $G_{K}$ induces an action of $G_{K} / G_{1} \subset \mathbf{Z}_{l}^{*}$ on the quotient group $G_{j} / G_{j+1}$. Moreover $G_{j} / G_{j+1}$ is isomorphic to a finite direct sum $\mathbf{Z}_{l}(j)^{n_{j}}$ as a $G_{K} / G_{1}$-module.

Proof. The corollary is a special case of Proposition 3.0.1 if $z=v$ and $p$ is a constant path.

The class of the element $\sigma \in H_{j}$ modulo $H_{j+1}$ is completely determined by its coordinates

$$
\begin{aligned}
& \left(\mathfrak{f}_{p}(\sigma), \mathfrak{f}_{\gamma_{1}}(\sigma), \ldots, \mathfrak{f}_{\gamma_{n}}(\sigma)\right) \\
& \quad \in\left(\Gamma^{j} \pi_{1}\left(X_{\bar{K}} ; v\right) / \Gamma^{j+1} \pi_{1}\left(X_{\bar{K}} ; v\right)\right) \times\left(\Gamma^{j} \pi_{1}\left(X_{\bar{K}} ; v\right) / \Gamma^{j+1} \pi_{1}\left(X_{\bar{K}} ; v\right)\right)^{n} .
\end{aligned}
$$

Apparentely the first coordinate $\mathfrak{f}_{p}(\sigma) \bmod \Gamma^{j+1} \pi_{1}\left(X_{\bar{K}} ; v\right)$ depends on a choice of a path $p$ from $v$ to $z$. However we have the following result.

LEMMA 3.0.3. Let $\sigma \in H_{j}$ and let $p$ and $q$ be two paths from $v$ to $z$. Then $\mathfrak{f}_{p}(\sigma) \equiv \mathfrak{f}_{q}(\sigma) \bmod \Gamma^{j+1} \pi_{1}\left(X_{\bar{K}} ; v\right)$.

Proof. Let us set $S=p^{-1} \cdot q$. Then $\mathfrak{f}_{q}(\sigma)=\mathfrak{f}_{p \cdot S}(\sigma)=S^{-1} \cdot \mathfrak{f}_{p}(\sigma) \cdot \sigma(S)$. Observe that $\sigma(S)=S \bmod \Gamma^{j+1} \pi_{1}\left(X_{\bar{K}} ; v\right)$. Hence we get that $\mathfrak{f}_{q}(\sigma)=$ $\mathfrak{f}_{p}(\sigma) \bmod \Gamma^{j+1} \pi_{1}\left(X_{\bar{K}} ; v\right)$.

It follows from Proposition 3.0.1 that $H_{k} / H_{i}$ are $l$-adic Lie groups. Let us set

$$
H_{\infty}=H_{\infty}(X ; z, v):=\bigcap_{i=1}^{\infty} H_{i}(X ; z, v) .
$$

Then $H_{1} / H_{\infty}=\lim _{i} H_{1} / H_{i}$ is a pro $l$-adic Lie group.

Definition 3.0.4. Let $A$ and $B$ be nilpotent groups with exponents in $\mathbf{Z}_{l}$. We say that a homomorphism $h: A \rightarrow B$ of groups with exponents in $\mathbf{Z}_{l}$ is an $f$-epimorphism if for any $b \in B$ there exists a positive integer $n$ and an element $a \in A$ such that $h(a)=b^{l^{n}}$.

Remark. If $A$ and $B$ are $\mathbf{Z}_{l}$-modules and if $B$ is a finitely generated $\mathbf{Z}_{l}$-module then $h: A \rightarrow B$ is an $f$-epimorphism if and only if $\operatorname{coker}(h)$ is finite. 


\section{Proposition 3.0.5. The natural homomorphisms}

$$
H_{i} / H_{k} \longrightarrow G_{i} / G_{k}
$$

are $f$-epimorphisms for any $i>0$ and any $k>0$ such that $k>i$.

Proof. The equality $H_{1}=G_{1}$ implies that the natural homomorphism $g: H_{1} / H_{k} \rightarrow G_{1} / G_{k}$ is an epimorphism for any $k$. After the Malcev rational completion we obtain an epimorphism $g_{0}: H_{1} / H_{k} \otimes \mathbf{Q} \rightarrow G_{1} / G_{k} \otimes \mathbf{Q}$ of nilpotent groups with exponents in $\mathbf{Q}_{l}$. The category of nilpotent groups with exponent in $\mathbf{Q}_{l}$ and the category of nilpotent Lie algebras over $\mathbf{Q}_{l}$ are equivalent. Hence passing to Lie algebras we get an epimorphism $\operatorname{Lie}\left(g_{0}\right)$ : $\operatorname{Lie}\left(H_{1} / H_{k} \otimes \mathbf{Q}\right) \rightarrow \operatorname{Lie}\left(G_{1} / G_{k} \otimes \mathbf{Q}\right)$ of finite dimensional nilpotent Lie algebras over $\mathbf{Q}_{l}$. The construction of the Malcev rational completion and then passing to Lie algebras are functorial. Therefore the Galois group $G_{K}$ acts linearly on both Lie algebras and the morphism $\operatorname{Lie}\left(g_{0}\right)$ is $G_{K^{-}}$ equivariant. Now the standard weight arguments imply that the natural morphism $\operatorname{Lie}\left(H_{i} / H_{k} \otimes \mathbf{Q}\right) \rightarrow \operatorname{Lie}\left(G_{i} / G_{k} \otimes \mathbf{Q}\right)$ is an epimorphism. Hence the homomorphism of nilpotent groups $H_{i} / H_{k} \otimes \mathbf{Q} \rightarrow G_{i} / G_{k} \otimes \mathbf{Q}$ is also an epimorphism. This implies that the natural map $H_{i} / H_{k} \rightarrow G_{i} / G_{k}$ is an $f$-epimorphism.

Let us set

$$
\mathcal{K}_{i}(X, v):=\bigcap_{z \in \hat{X}(K)} H_{i}(X ; z, v), \quad \mathcal{K}_{i}(X):=\bigcap_{(z, v) \in \hat{X}(K)^{2}} H_{i}(X ; z, v)
$$

and

$$
\mathcal{K}_{\infty}(X, v):=\bigcap_{i=1}^{\infty} \mathcal{K}_{i}(X, v), \quad \mathcal{K}_{\infty}(X):=\bigcap_{i=1}^{\infty} \mathcal{K}_{i}(X)
$$

3.0.6. Observe that $\mathcal{K}_{1}(X)=\mathcal{K}_{1}(X, v)=G_{1}(X, v)=\operatorname{Gal}\left(\bar{K} / K\left(\mu_{l^{\infty}}\right)\right)$. We do not know if the maps

$$
\mathcal{K}_{i}(X, v) / \mathcal{K}_{k}(X, v) \longrightarrow H_{i}(X ; z, v) / H_{k}(X ; z, v)
$$

and

$$
\mathcal{K}_{i}(X) / \mathcal{K}_{k}(X) \longrightarrow H_{i}(X ; z, v) / H_{k}(X ; z, v)
$$

are $f$-epimorphisms for any $i$ and any $k$. Below we shall show weaker results. 
Let $T$ be a nonempty finite subset of $\hat{X}(K)^{2}$. Let us set

$$
\mathcal{K}_{i}^{T}(X):=\bigcap_{(z, v) \in T} H_{i}(X ; z, v) \text { and } \mathcal{K}_{\infty}^{T}(X):=\bigcap_{i=1}^{\infty} \mathcal{K}_{i}^{T}(X) .
$$

In the same way as Proposition 3.0.5 we show the following result.

Proposition 3.0.7. Let $T$ and $S$ be nonempty finite subsets of $\hat{X}(K)^{2}$. Assume that $S \subset T$. Then the maps

$$
\mathcal{K}_{i}^{T}(X) / \mathcal{K}_{k}^{T}(X) \longrightarrow \mathcal{K}_{i}^{S}(X) / \mathcal{K}_{k}^{S}(X)
$$

are $f$-epimorphisms for any positive integers $k$ and $i$ such that $k>i$.

LEMMA 3.0.8. The restriction map

$$
H^{1}\left(G_{K}, \mathbf{Q}_{l}(N)\right) \longrightarrow H^{1}\left(\mathcal{K}_{N}^{T}(X), \mathbf{Q}_{l}(N)\right)
$$

is injective.

Proof. Let $\Gamma=\operatorname{Gal}\left(K\left(\mu_{l \infty}\right) / K\right)$. We recall the reader that $\mathcal{K}_{1}^{T}(X)=$ $\operatorname{Gal}\left(\bar{K} / K\left(\mu_{l}\right)\right)$. The restriction map

$$
H^{1}\left(G_{K}, \mathbf{Q}_{l}(N)\right) \longrightarrow \operatorname{Hom}_{\Gamma}\left(\mathcal{K}_{1}^{T}(X)^{a b}, \mathbf{Q}_{l}(N)\right)
$$

is injective. Let $f \in \operatorname{Hom}_{\Gamma}\left(\mathcal{K}_{1}^{T}(X)^{a b}, \mathbf{Q}_{l}(N)\right)$. Assume that the composition of $f$ with the natural projection $\mathcal{K}_{1}^{T}(X) \rightarrow \mathcal{K}_{1}^{T}(X)^{a b}$ vanishes on $\mathcal{K}_{N}^{T}(X)$. Therefore $f$ induces a $\Gamma$-homomorphism $\tilde{f}:\left(\mathcal{K}_{1}^{T}(X) / \mathcal{K}_{N}^{T}(X)\right)^{a b} \rightarrow \mathbf{Q}_{l}(N)$. Proposition 3.0.1 implies that the quotient group $\mathcal{K}_{1}^{T}(X) / \mathcal{K}_{N}^{T}(X)$ is a successive extension of direct sums of $\mathbf{Z}_{l}(i)$ with $i<N$. Now it follows from weight arguments that $\tilde{f}$ and hence also $f$ are zero maps. This implies the lemma.

DEFinition 3.0.9. Let $\mathcal{C}$ be the category whose objects are all finite subsets of $\hat{X}(K)^{2}$ and whose morphisms are inclusions. We set

$$
H_{\mathcal{C}}^{1}\left(\mathcal{K}_{N}(X), \mathbf{Q}_{l}(N)\right):=\underset{\mathcal{C}}{\lim } H^{1}\left(\mathcal{K}_{N}^{T}(X), \mathbf{Q}_{l}(N)\right) .
$$

LEMMA 3.0.10. The map

$$
H^{1}\left(G_{K}, \mathbf{Q}_{l}(N)\right) \longrightarrow H_{\mathcal{C}}^{1}\left(\mathcal{K}_{N}(X), \mathbf{Q}_{l}(N)\right)
$$

is injective. 
Proof. The lemma follows from Lemma 3.0.8.

Lemma 3.0.10 will be needed in our formulation of Zagier conjecture in Section 7. We recall also that $H^{1}\left(G_{K}, \mathbf{Q}_{l}(N)\right)$ for $N>1$ is a finite dimensional vector space over $\mathbf{Q}_{l}$. More precisely there is the following result. Let $r_{1}$ (resp. $r_{2}$ ) be a number of real (resp. complex) places of $K$. We assume that $l$ is an odd prime. Let $S$ be a set of maximal ideals of $\mathcal{O}_{K}$ containing all maximal ideals which divide $l$ and let $\mathcal{O}_{K, S}$ be a ring of $S$-integers in $K$. Then

$$
\begin{aligned}
\operatorname{dim} H^{1}\left(\operatorname{Spec} \mathcal{O}_{K, S}, \mathbf{Q}_{l}(N)\right)= & \operatorname{dim} H^{1}\left(G_{K}, \mathbf{Q}_{l}(N)\right)=r_{2}, \\
& \text { if } N \text { is even and greater than } 1 ; \\
\operatorname{dim} H^{1}\left(\operatorname{Spec} \mathcal{O}_{K, S}, \mathbf{Q}_{l}(N)\right)= & \operatorname{dim} H^{1}\left(G_{K}, \mathbf{Q}_{l}(N)\right)=r_{1}+r_{2}, \\
& \text { if } N \text { is odd and greater than } 1 .
\end{aligned}
$$

(See [S2, Theorem 1] for $\mathcal{O}_{K}\left[\frac{1}{l}\right]$ and apply Proposition 1 from [S1] for $K$ and $\mathcal{O}_{K, S}$.)

Let us assume that $\mathcal{O}_{K, S}^{*} \otimes \mathbf{Q}$ is a finite dimensional vector space over Q. Then

$$
\operatorname{dim} H^{1}\left(\operatorname{Spec} \mathcal{O}_{K, S}, \mathbf{Q}_{l}(1)\right)=\operatorname{dim}_{\mathbf{Q}}\left(\mathcal{O}_{K, S}^{*} \otimes \mathbf{Q}\right) .
$$

The last equality follows from Kummer theory.

3.1. We shall study relations between filtrations $\left\{G_{i}\right\}_{i \in \mathbf{N}}$ and $\left\{H_{i}\right\}_{i \in \mathbf{N}}$ of $G_{K}$ for different $X$.

Lemma 3.1.0. Let $Y=P_{K}^{1} \backslash\left\{b_{1}, \ldots, b_{m+1}\right\}$ and let $g: Y \rightarrow X$ be a non-constant morphism between affine varieties. Let $y, w \in \hat{Y}(K)$ and let $z=g(y)$ and $v=g(w)$. Then we have $G_{i}(Y, w) \subset G_{i}(X, v)$ and $H_{i}(Y ; y, w) \subset H_{i}(X ; z, v)$.

Proof. Observe that the induced map $g_{*}: \pi_{1}\left(Y_{\bar{K}} ; w\right) \rightarrow \pi_{1}\left(X_{\bar{K}} ; v\right)$ is surjective after passing to the Malcev rational completions and it commutes with the action of $G_{K}$. This implies that $G_{i}(Y, w) \subset G_{i}(X, v)$. Let $p$ be a path from $w$ to $y$. Then $\mathfrak{f}_{g(p)}(\sigma)=g_{*}\left(\mathfrak{f}_{p}(\sigma)\right)$. Hence $\mathfrak{f}_{p}(\sigma) \in \Gamma^{i} \pi_{1}\left(Y_{\bar{K}} ; w\right)$ implies that $\mathfrak{f}_{g(p)}(\sigma) \in \Gamma^{i} \pi_{1}\left(X_{\bar{K}} ; v\right)$. This implies that $H_{i}(Y ; y, w) \subset$ $H_{i}(X ; z, v)$.

As before the weight arguments imply the following result. 
Proposition 3.1.1. The induced maps

$$
G_{i}(Y, w) / G_{i+k}(Y, w) \longrightarrow G_{i}(X, v) / G_{i+k}(X, v)
$$

and

$$
H_{i}(Y ; y, w) / H_{i+k}(Y ; y, w) \longrightarrow H_{i}(X ; z, v) / H_{i+k}(X ; z, v)
$$

are $f$-epimorphisms for all $i>0$ and all $k>0$.

3.2. We recall that $x=\left(x_{1}, x_{2}, \ldots, x_{n+1}\right)$ is a sequence of geometric generators of $\pi_{1}(X(\mathbf{C}) ; v)$. Then $\pi_{1}\left(X_{\bar{K}} ; v\right)$ is a free pro-l group on $n$ generators $x_{1}, \ldots, x_{n}$. Let $\operatorname{Lie}(\mathbf{X})$ be a free Lie algebra on $n$ generators $X_{1}, \ldots, X_{n}$. Let us fix a Hall base $\mathcal{B}$ of $\operatorname{Lie}(\mathbf{X})$. Let $\mathcal{B}_{i}$ be the set of elements of degree $i$ in $\mathcal{B}$. We introduce a linear order in the set $\mathcal{B}$ in the following way. We fix a linear order in $\mathcal{B}_{i}$ for every $i$. We assume that elements of $\mathcal{B}_{i}$ are smaller than elements of $\mathcal{B}_{i+1}$.

If $e=\left[\cdots\left[X_{i_{1}}, X_{i_{2}}\right] X_{i_{3}} \cdots\right]$, we denote by $e(x)$ the element $\left(\cdots\left(x_{i_{1}}, x_{i_{2}}\right)\right.$ $\left.x_{i_{3}} \cdots\right)$ of $\pi_{1}\left(X_{\bar{K}} ; v\right)$. It is well known that any $g \in \pi_{1}\left(X_{\bar{K}} ; v\right)$ can be written uniquely as an infinite convergent product

$$
\prod_{i=1}^{\infty} \prod_{e \in \mathcal{B}_{i}} e(x)^{\alpha_{e}}
$$

where $\alpha_{e} \in \mathbf{Z}_{l}$ and the product is taken in the declared linear order in $\mathcal{B}$.

Definition 3.2.0. Let $z \in \hat{X}(K)$ and let $p \in \pi\left(X_{\bar{K}} ; z, v\right)$. For each $e \in \mathcal{B}_{j}$ we define maps

$$
\kappa_{e}(p, x): H_{j}(X ; z, v) \longrightarrow \mathbf{Z}_{l}(j)
$$

by the following equations

$$
\mathfrak{f}_{p}(\sigma) \equiv \prod_{e \in \mathcal{B}_{j}} e(x)^{\kappa_{e}(p, x)(\sigma)} \bmod \Gamma^{j+1} \pi_{1}\left(X_{\bar{K}} ; v\right) .
$$

Lemma 3.2.1. Let $z \in \hat{X}(K)$ and let $p \in \pi\left(X_{\bar{K}} ; z, v\right)$. Let $e \in \mathcal{B}_{j}$. The map $\kappa_{e}(p, x): H_{j}(X ; z, v) \rightarrow \mathbf{Z}_{l}(j)$ is a homomorphism compatible with actions of $\operatorname{Gal}\left(K\left(\mu_{l}\right) / K\right)$. The map $\kappa_{e}(p, x)$ does not depend on the choice of a path $p$ from $v$ to $z$ and it does not depend on the choice of a sequence of geometric generators $x=\left(x_{1}, x_{2}, \ldots, x_{n+1}\right)$ in the same permutation class. 
Proof. Let $x^{\prime}=\left(x_{1}^{\prime}, \ldots, x_{n+1}^{\prime}\right)$ be another sequence of geometric generators of $\pi_{1}(X(\mathbf{C}) ; v)$ associated with a family of paths $\Gamma^{\prime}=\left\{\gamma_{i}^{\prime}\right\}_{i=1, \ldots, n+1}$. We shall assume that the automorphism of $\pi_{1}(X(\mathbf{C}) ; v)$ given by $x_{i} \rightarrow x_{i}^{\prime}$ for $i=1, \ldots, n+1$ is in $\mathcal{B}_{n+1}^{(1) *}\left(x_{1}, \ldots, x_{n+1}\right)$. We have

$$
x_{i}^{\prime}=f_{i}\left(x_{1}, \ldots, x_{n}\right)^{-1} \cdot x_{i} \cdot f_{i}\left(x_{1}, \ldots, x_{n}\right) \quad(i=1, \ldots, n+1),
$$

where $f_{i}\left(x_{1}, \ldots, x_{n}\right):=\gamma_{i}^{-1} \cdot \gamma_{i}^{\prime} \in \pi_{1}(X(\mathbf{C}) ; v)$. Then

$$
\mathfrak{f}_{p}(\sigma) \equiv \prod_{e \in \mathcal{B}_{j}} e\left(x^{\prime}\right)^{\kappa_{e}\left(p, x^{\prime}\right)(\sigma)} \bmod \Gamma^{j+1} \pi_{1}\left(X_{\bar{K}} ; v\right) .
$$

It follows from (3.2.2) that $e(x) \equiv e\left(x^{\prime}\right) \bmod \Gamma^{j+1} \pi_{1}\left(X_{\bar{K}} ; v\right)$. Hence $\kappa_{e}(p, x)=\kappa_{e}\left(p, x^{\prime}\right)$.

Let $q \in \pi\left(X_{\bar{K}} ; z, v\right)$ and let $T:=p^{-1} \cdot q$. Then it follows from (1.0.4) that

$$
\mathfrak{f}_{q}(\sigma)=T^{-1} \cdot \mathfrak{f}_{p}(\sigma) \cdot \sigma(T) .
$$

If $\sigma \in G_{j}(X, v)$ then $\sigma(T)=T \bmod \Gamma^{j+1} \pi_{1}\left(X_{\bar{K}} ; v\right)$. Hence we get $\mathfrak{f}_{q}(\sigma)=$ $T^{-1} \cdot \mathfrak{f}_{p}(\sigma) \cdot \sigma(T)=\mathfrak{f}_{p}(\sigma) \bmod \Gamma^{j+1} \pi_{1}\left(X_{\bar{K}} ; v\right)$. Therefore $\kappa_{e}(p, x)$ does not depend on the choice of a path $p$ in $\pi\left(X_{\bar{K}} ; z, v\right)$.

The formula

$$
\mathfrak{f}_{p}(\tau \sigma)=\mathfrak{f}_{p}(\tau) \cdot \tau\left(\mathfrak{f}_{p}(\sigma)\right)
$$

(see Proposition 1.0.7) and Proposition 2.2.1 imply that $\kappa_{e}(p, x)$ is a homomorphism.

Let $\tau \in G_{K}$ and $\sigma \in H_{j}(X ; z, v)$. Then $\tau \sigma \tau^{-1} \in H_{j}(X ; z, v)$ and

$$
\mathfrak{f}_{p}\left(\tau \sigma \tau^{-1}\right) \equiv \prod_{e \in \mathcal{B}_{j}} e(x)^{\kappa_{e}(p, x)\left(\tau \sigma \tau^{-1}\right)} \bmod \Gamma^{j+1} \pi_{1}\left(X_{\bar{K}} ; v\right) .
$$

On the other hand

$$
\mathfrak{f}_{p}\left(\tau \sigma \tau^{-1}\right)=\mathfrak{f}_{p}(\tau) \cdot \tau\left(\mathfrak{f}_{p}(\sigma)\right) \cdot \tau \sigma\left(\mathfrak{f}_{p}\left(\tau^{-1}\right)\right) .
$$

Working $\bmod \Gamma^{j+1} \pi_{1}\left(X_{\bar{K}} ; v\right)$ we get

$$
\begin{aligned}
\mathfrak{f}_{p}(\tau) \cdot \tau\left(\mathfrak{f}_{p}(\sigma)\right) \cdot \tau \sigma\left(\mathfrak{f}_{p}\left(\tau^{-1}\right)\right) & \equiv \mathfrak{f}_{p}(\tau) \cdot \prod_{e \in \mathcal{B}_{j}} e(x)^{\chi(\tau)^{j} \kappa_{e}(p, x)(\sigma)} \cdot \tau\left(\mathfrak{f}_{p}\left(\tau^{-1}\right)\right) \\
& \equiv \prod_{e \in \mathcal{B}_{j}} e(x)^{\chi(\tau)^{j} \kappa_{e}(p, x)(\sigma)}
\end{aligned}
$$


because $\sigma\left(\mathfrak{f}_{p}\left(\tau^{-1}\right)\right) \equiv \mathfrak{f}_{p}\left(\tau^{-1}\right) \bmod \Gamma^{j+1} \pi_{1}\left(X_{\bar{K}} ; v\right)$ and $\tau\left(\mathfrak{f}_{p}\left(\tau^{-1}\right)\right)=$ $\left(\mathfrak{f}_{p}(\tau)\right)^{-1}$. Hence we get that $\kappa_{e}(p, x)\left(\tau \sigma \tau^{-1}\right)=\chi(\tau)^{j} \kappa_{e}(p, x)(\sigma)$.

Observe that the homomorphism $\kappa_{e}(p, x): H_{j}(X ; z, v) \rightarrow \mathbf{Z}_{l}(j)$ depends only on $(z, v) \in \hat{X}(K)^{2}$ and on a linear order $\left(a_{1}, \ldots, a_{n+1}\right)$ of points removed from $\mathbf{P}_{K}^{1}$. Assuming that the linear order $\left(a_{1}, \ldots, a_{n+1}\right)$ is fixed we set

$$
\kappa_{e}(z, v):=\kappa_{e}(p, x)
$$

\section{$\S 4$. Coordinates on the fundamental group and on the torsor}

4.0. Let $X=\mathbf{P}_{K}^{1} \backslash\left\{a_{1}, \ldots, a_{n+1}\right\}$ and let $v \in \hat{X}(K)$. Let $x=$ $\left(x_{1}, \ldots, x_{n+1}\right)$ be a sequence of geometric generators of $\pi_{1}(X(\mathbf{C}) ; v)$. Let $\mathbf{Q}_{l}\left\{\left\{X_{1}, \ldots, X_{n}\right\}\right\}$ be an algebra of non-commutative formal power series in $n$ non-commuting variables $X_{1}, \ldots, X_{n}$. We set $\mathbf{X}:=\left\{X_{1}, \ldots, X_{n}\right\}$. To simplify the notation we shall write $\mathbf{Q}_{l}\{\{\mathbf{X}\}\}$ instead of $\mathbf{Q}_{l}\left\{\left\{X_{1}, \ldots, X_{n}\right\}\right\}$.

We recall that $\mathbf{Q}_{l}$ is a topological non-archimedian field. Let $I$ be the augmentation ideal of $\mathbf{Q}_{l}\{\{\mathbf{X}\}\}$. Observe that $\mathbf{Q}_{l}\{\{\mathbf{X}\}\} / I^{m}$ is a finite dimensional topological vector space over $\mathbf{Q}_{l}$ and $\mathbf{Q}_{l}\{\{\mathbf{X}\}\}=\lim _{m} \mathbf{Q}_{l}\{\{\mathbf{X}\}\} / I^{m}$. We equip $\mathbf{Q}_{l}\{\{\mathbf{X}\}\}$ with a topology of the projective limit. We recall that $\pi_{1}\left(X_{\bar{K}} ; v\right)$ is equipped with a pro-finite topology.

We define a continuous embedding

$$
k_{x}: \pi_{1}\left(X_{\bar{K}} ; v\right) \longrightarrow \mathbf{Q}_{l}\{\{\mathbf{X}\}\}
$$

setting $k_{x}\left(x_{i}\right):=\exp X_{i}$ for $i=1, \ldots, n$ and requiring that $k_{x}\left(w \cdot w^{\prime}\right)=$ $k_{x}(w) \cdot k_{x}\left(w^{\prime}\right)$.

Let $p \in \pi\left(X_{\bar{K}} ; z, v\right)$. Composing $t_{p}$ (see Section 1) with $k_{x}$ we get a continuous embedding

$$
k_{x, p}: \pi\left(X_{\bar{K}} ; z, v\right) \longrightarrow \mathbf{Q}_{l}\{\{\mathbf{X}\}\}
$$

Let us set

$$
\Lambda_{(p, x)}(\sigma):=k_{x}\left(\mathfrak{f}_{p}(\sigma)\right) .
$$

(We shall omit the subscript $x$ if a sequence of geometric generators is fixed and we shall write $\Lambda_{p}(\sigma)$ instead of $\Lambda_{(p, x)}(\sigma)$.)

Let us denote by $\operatorname{Aut}\left(\mathbf{Q}_{l}\{\{\mathbf{X}\}\}\right)$ the group of continuous automorphisms of the $\mathbf{Q}_{l}$-algebra $\mathbf{Q}_{l}\{\{\mathbf{X}\}\}$ and by $\operatorname{GL}\left(\mathbf{Q}_{l}\{\{\mathbf{X}\}\}\right)$ the group of continuous linear automorphisms of the $\mathbf{Q}_{l}$-vector space $\mathbf{Q}_{l}\{\{\mathbf{X}\}\}$. 
The action of $G_{K}$ on $\pi_{1}\left(X_{\bar{K}} ; v\right)$ defines a continuous action of $G_{K}$ on $\mathbf{Q}_{l}\{\{\mathbf{X}\}\}$,

$$
()_{x}: G_{K} \longrightarrow \operatorname{Aut}\left(\mathbf{Q}_{l}\{\{\mathbf{X}\}\}\right)
$$

given by $\sigma_{x}\left(\exp X_{i}\right):=k_{x}\left(\sigma\left(x_{i}\right)\right)$ for $i=1, \ldots, n$.

The action of $G_{K}$ on $\pi\left(X_{\bar{K}} ; z, v\right)$ defines a continuous action of $G_{K}$ on $\mathbf{Q}_{l}\{\{\mathbf{X}\}\}$,

$$
()_{x, p}: G_{K} \longrightarrow \mathrm{GL}\left(\mathbf{Q}_{l}\{\{\mathbf{X}\}\}\right)
$$

given by $\sigma_{x, p}(w):=\Lambda_{(p, x)}(\sigma) \cdot \sigma_{x}(w)$.

(We shall omit the subscript $x$ if a sequence of geometric generators is fixed and we shall write $\sigma$ instead of $\sigma_{x}$ and $\sigma_{p}$ instead of $\sigma_{x, p}$. We hope that these notations will not cause confusions with notations used in Section 1. There $\sigma$ (resp. $\sigma_{p}$ ) denotes an automorphism of $\pi_{1}(X(\mathbf{C}) ; v)$ (resp. a bijection of $\left.\pi_{1}(X(\mathbf{C}) ; v)\right)$ induced from the action of $G_{K}$ on $\pi_{1}(X(\mathbf{C}) ; v)$ (resp. on the $\pi_{1}(X(\mathbf{C}) ; v)$-torsor $\left.\pi\left(X_{\bar{K}} ; z, v\right)\right)$.)

4.1. The subgroups $G_{i}(X, v)$ and $H_{i}(X ; z, v)$ of $G_{K}$ can be described in terms of the action of $G_{K}$ on $\mathbf{Q}_{l}\{\{\mathbf{X}\}\}$ in the following way.

Lemma 4.1.1. Let $X=\mathbf{P}_{K}^{1} \backslash\left\{a_{1}, \ldots, a_{n+1}\right\}$ and let $z, v \in \hat{X}(K)$. We have

$$
G_{i}(X ; v)=\operatorname{ker}\left(G_{K} \rightarrow \operatorname{Aut}\left(\mathbf{Q}_{l}\{\{\mathbf{X}\}\} / I^{i+1}\right)\right)
$$

and

$$
H_{i}(X ; z, v)=\operatorname{ker}\left(G_{i}(X ; v) \rightarrow \operatorname{GL}\left(\mathbf{Q}_{l}\{\{\mathbf{X}\}\} / I^{i}\right)\right) .
$$

We shall omit an easy proof.

4.2. Let $\lambda \in \mathbf{Q}_{l}^{*}$. We define a continuous automorphism of $\mathbf{Q}_{l}$-algebras

$$
\rho(\lambda): \mathbf{Q}_{l}\{\{\mathbf{X}\}\} \longrightarrow \mathbf{Q}_{l}\{\{\mathbf{X}\}\}
$$

setting $\rho(\lambda)(w):=\lambda^{i} w$ if $w$ is homogenous of degree $i$.

Let $\sigma \in G_{K}$. We set

$$
\varphi_{x}(\sigma):=\sigma_{x} \circ \rho\left(\chi(\sigma)^{-1}\right)
$$

and

$$
\psi_{x, p}(\sigma):=\sigma_{x, p} \circ \rho\left(\chi(\sigma)^{-1}\right) .
$$

Observe that $\varphi_{x}(\sigma)$ (resp. $\left.\psi_{x, p}(\sigma)\right)$ is a pro-unipotent automorphism of $\mathbf{Q}_{l^{-}}$ algebra (resp. pro-unipotent $\mathbf{Q}_{l}$-linear automorphism of) $\mathbf{Q}_{l}\{\{\mathbf{X}\}\}$. 
Remark. If $\sigma \in G_{1}$ then $\varphi_{x}(\sigma)=\sigma_{x}$ and $\psi_{x, p}(\sigma)=\sigma_{x, p}$.

LEMmA 4.2.1. We have

$$
\varphi_{x}(\tau \cdot \sigma)=\varphi_{x}(\tau) \circ\left(\rho(\chi(\tau)) \circ \varphi_{x}(\sigma) \circ \rho\left(\chi(\tau)^{-1}\right)\right)
$$

and

$$
\psi_{x, p}(\tau \cdot \sigma)=\psi_{x, p}(\tau) \circ\left(\rho(\chi(\tau)) \circ \psi_{x, p}(\sigma) \circ \rho\left(\chi(\tau)^{-1}\right)\right) .
$$

We can interpret the equalities from Lemma 4.2.1 in the following way.

Corollary 4.2.2. Let $G_{K}$ acts on $\operatorname{Aut}\left(\mathbf{Q}_{l}\{\{\mathbf{X}\}\}\right)$ (resp. $\left.\mathrm{GL}\left(\mathbf{Q}_{l}\{\{\mathbf{X}\}\}\right)\right)$ by $\sigma(a):=\rho(\chi(\sigma)) \circ a \circ \rho\left(\chi(\sigma)^{-1}\right)$. Then the maps $\varphi_{x}: G_{K} \rightarrow \operatorname{Aut}\left(\mathbf{Q}_{l}\{\{\mathbf{X}\}\}\right)$ and $\psi_{x, p}: G_{K} \rightarrow \operatorname{Aut}\left(\mathbf{Q}_{l}\{\{\mathbf{X}\}\}\right)$ are 1-cocycles.

Let $\lambda \in \mathbf{Q}_{l}^{*}$. We shall denote by $a^{\lambda}$ the automorphism $\rho\left(\lambda^{-1}\right) \circ a \circ \rho(\lambda)$

\section{$\S 5 . \quad l$-adic iterated integrals}

5.0. The purpose of this section is to introduce objects called by us $l$ adic iterated integrals (see Definition 5.3.0). These $l$-adic iterated integrals evaluated at $z$ are functions from the Galois group $G_{K}$ to $\mathbf{Q}_{l}$, which to $\sigma \in$ $G_{K}$ associate coefficients of the power series $\left(\log \psi_{x, p}(\sigma)\right)(1)\left(\left(\log \sigma_{x, p}\right)(1)\right.$ if $\left.\sigma \in G_{K\left(\mu_{l} \infty\right)}\right)$. These $l$-adic iterated integrals correspond to suitably normalized classical complex iterated integrals.

Let $a_{1}, \ldots, a_{n+1}$ be $K$-points of the projective line $\mathbf{P}_{K}^{1}$. Let $X=\mathbf{P}_{K}^{1} \backslash$ $\left\{a_{1}, \ldots, a_{n+1}\right\}$ and let $v \in \hat{X}(K)$ be a base point. Let us choose a tangential base point $v_{i}$ at $a_{i}$ for $i=1,2, \ldots, n+1$. Let $x=\left(x_{1}, \ldots, x_{n+1}\right)$ be a sequence of geometric generators of $\pi_{1}(X(\mathbf{C}) ; v)$ associated with a family of paths $\Gamma=\left\{\gamma_{i}\right\}_{i=1, \ldots, n+1}$ from $v$ to each $v_{i}$. It follows from Section 3 that $G_{1} / G_{\infty}$ is a pro-unipotent $l$-adic Lie group. Hence $\left(G_{1} / G_{\infty}\right) \otimes \mathbf{Q}$ - the rational completion of $G_{1} / G_{\infty}$ - is a pro-unipotent $\mathbf{Q}_{l}$-Lie group. Let us set $\mathfrak{g}=\mathfrak{g}(X, v):=T_{i d}\left(\left(G_{1} / G_{\infty}\right) \otimes \mathbf{Q}\right)=\operatorname{Lie}\left(\left(G_{1} / G_{\infty}\right) \otimes \mathbf{Q}\right)$ - the tangent space of $\left(G_{1} / G_{\infty}\right) \otimes \mathbf{Q}$ at the identity.

We shall denote by $\operatorname{Der}\left(\mathbf{Q}_{l}\{\{\mathbf{X}\}\}\right)$ the Lie algebra of continuous derivations of the $\mathbf{Q}_{l}$-algebra $\mathbf{Q}_{l}\{\{\mathbf{X}\}\}$ and by $\operatorname{End}\left(\mathbf{Q}_{l}\{\{\mathbf{X}\}\}\right)$ the Lie algebra of continuous automorphisms of the $\mathbf{Q}_{l}$-vector space $\mathbf{Q}_{l}\{\{\mathbf{X}\}\}$. 
We have the following commutative diagram:

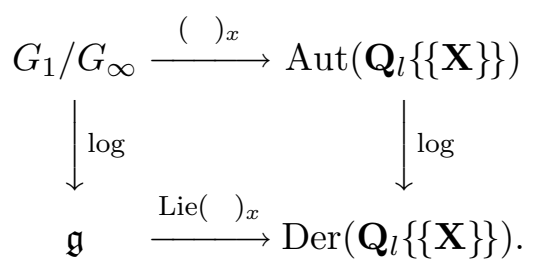

(The upper horizontal arrow is induced by the action of $G_{K}$ on $\mathbf{Q}_{l}\{\{\mathbf{X}\}\}$, the lower horizontal arrow is the induced map on tangent spaces, log on the right side is defined only on pro-unipotent automorphisms.)

Let $z \in \hat{X}(K)$ and let $p \in \pi\left(X_{\bar{K}} ; z, v\right)$. It follows from Section 3 that $H_{1} / H_{\infty}=H_{1}(X ; z, v) / H_{\infty}(X ; z, v)$ is a pro-unipotent $l$-adic Lie group. Hence $\left(H_{1} / H_{\infty}\right) \otimes \mathbf{Q}$ is a pro-unipotent $\mathbf{Q}_{l}$-Lie group. Let us set $\mathfrak{h}=$ $\mathfrak{h}(X ; z, v):=T_{i d}\left(\left(H_{1} / H_{\infty}\right) \otimes \mathbf{Q}\right)=\operatorname{Lie}\left(\left(H_{1} / H_{\infty}\right) \otimes \mathbf{Q}\right)$ - the tangent space of $\left(H_{1} / H_{\infty}\right) \otimes \mathbf{Q}$ at the identity. We have the following commutative diagram:

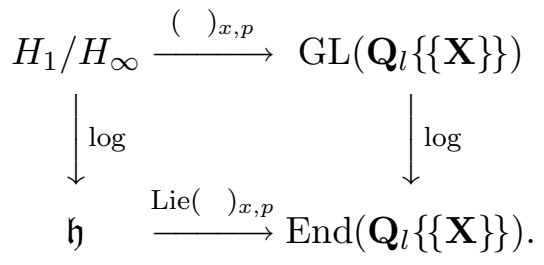

Let $T \subset \hat{X}(K)^{2}$ be a finite subset containing a pair $(z, v)$. We have epimorphisms $\mathcal{K}_{1}^{T}(X) / \mathcal{K}_{\infty}^{T}(X) \rightarrow G_{1} / G_{\infty}$ and $\mathcal{K}_{1}^{T}(X) / \mathcal{K}_{\infty}^{T}(X) \rightarrow H_{1} / H_{\infty}$ and the induced epimorphisms of Lie algebras

$$
\operatorname{Lie}\left(\mathcal{K}_{1}^{T}(X) / \mathcal{K}_{\infty}^{T}(X) \otimes \mathbf{Q}\right) \longrightarrow \mathfrak{g} \quad \text { and } \quad \operatorname{Lie}\left(\mathcal{K}_{1}^{T}(X) / \mathcal{K}_{\infty}^{T}(X) \otimes \mathbf{Q}\right) \longrightarrow \mathfrak{h}
$$

Hence we can consider that the homomorphisms ()$_{x}$ and ()$_{x, p}$ are defined on $\mathcal{K}_{1}^{T}(X) / \mathcal{K}_{\infty}^{T}(X)$ and that the morphisms of Lie algebras $\operatorname{Lie}()_{x}$ and $\operatorname{Lie}()_{x, p}$ are defined on $\operatorname{Lie}\left(\mathcal{K}_{1}^{T}(X) / \mathcal{K}_{\infty}^{T}(X) \otimes \mathbf{Q}\right)$.

The image of the morphism ()$_{x}$ (resp. $\left.\operatorname{Lie}()_{x}\right)$ is contained in the "braid-like" subgroup of $\operatorname{Aut}\left(\mathbf{Q}_{l}\{\{\mathbf{X}\}\}\right.$ ) (resp. Lie subalgebra of Der $\left.\left(\mathbf{Q}_{l}\{\{\mathbf{X}\}\}\right)\right)$. We recall their definitions. We also describe subgroups and subalgebras containing images of morphisms ()$_{x, p}$ and $\operatorname{Lie}()_{x, p}$ respectively. 
5.1. We recall that $\mathbf{X}=\left\{X_{1}, \ldots, X_{2}\right\}$. Let $\operatorname{Lie}(\mathbf{X})$ be a free Lie algebra over $\mathbf{Q}_{l}$ on the set $\mathbf{X}$. Let us set

$$
L(\mathbf{X}):={\underset{\iota}{i}}_{i} \operatorname{Lie}(\mathbf{X}) / \Gamma^{i} \operatorname{Lie}(\mathbf{X})
$$

We identify $L(\mathbf{X})$ with Lie elements in $\mathbf{Q}_{l}\{\{\mathbf{X}\}\}$.

We introduce the following notation. If $A$ and $B$ belong to a Lie algebra then we define $\left[[A, B] B^{0}\right]:=[A, B],\left[[A, B] B^{1}\right]:=[[A, B], B]$ and $\left[[A, B] B^{m}\right]:=\left[\left[[A, B] B^{m-1}\right], B\right]$ for $m>1$.

Definition 5.1.0. Let us define subgroups

$$
\begin{aligned}
& \operatorname{Aut}^{*}\left(\mathbf{Q}_{l}\{\{\mathbf{X}\}\}\right):=\left\{f \in \operatorname{Aut}\left(\mathbf{Q}_{l}\{\{\mathbf{X}\}\}\right) \mid\right. \\
& \left.\qquad X_{i} \in \mathbf{X} \exists l_{i} \in L(\mathbf{X}), f\left(X_{i}\right)=e^{-l_{i}} \cdot X_{i} \cdot e^{l_{i}}\right\} ; \\
& \text { Aut }^{*} L(\mathbf{X}):=\{f \in \operatorname{Aut} L(\mathbf{X}) \mid \\
& \left.\forall X_{i} \in \mathbf{X} \exists l_{i} \in L(\mathbf{X}), f\left(X_{i}\right)=X_{i}+\sum_{m=1}^{\infty} \frac{1}{m !}\left[\left[X_{i}, l_{i}\right] l_{i}^{m-1}\right]\right\}
\end{aligned}
$$

and Lie subalgebras

$$
\begin{aligned}
& \operatorname{Der}^{*}\left(\mathbf{Q}_{l}\{\{\mathbf{X}\}\}\right):=\left\{D \in \operatorname{Der}\left(\mathbf{Q}_{l}\{\{\mathbf{X}\}\}\right) \mid\right. \\
& \left.\forall X_{i} \in \mathbf{X} \exists A_{i} \in L(\mathbf{X}), D\left(X_{i}\right)=X_{i} \cdot A_{i}-A_{i} \cdot X_{i}\right\} ; \\
& \operatorname{Der}^{*} L(\mathbf{X}):=\left\{D \in \operatorname{Der} L(\mathbf{X}) \mid \forall X_{i} \in \mathbf{X} \exists A_{i} \in L(\mathbf{X}), D\left(X_{i}\right)=\left[X_{i}, A_{i}\right]\right\}
\end{aligned}
$$

and

$$
\begin{aligned}
\operatorname{Der}^{*} \operatorname{Lie}(\mathbf{X}):=\{D \in \operatorname{Der} \operatorname{Lie}(\mathbf{X}) \mid & \\
& \left.\forall X_{i} \in \mathbf{X} \exists A_{i} \in \operatorname{Lie}(\mathbf{X}), D\left(X_{i}\right)=\left[X_{i}, A_{i}\right]\right\} .
\end{aligned}
$$

LEMma 5.1.1. We have

i) $\operatorname{Aut}^{*}\left(\mathbf{Q}_{l}\{\{\mathbf{X}\}\}\right)=\operatorname{Aut}^{*} L(\mathbf{X})$;

ii) $\operatorname{Der}^{*}\left(\mathbf{Q}_{l}\{\{\mathbf{X}\}\}\right)=\operatorname{Der}^{*} L(\mathbf{X})$;

iii) The Lie algebra of $\operatorname{Aut}^{*}\left(\mathbf{Q}_{l}\{\{\mathbf{X}\}\}\right)$ (resp. Aut $\left.{ }^{*}(\mathbf{X})\right)$ is $\operatorname{Der}^{*}\left(\mathbf{Q}_{l}\{\{\mathbf{X}\}\}\right)\left(\operatorname{resp} . \operatorname{Der}^{*} L(\mathbf{X})\right)$. 
Proof. The first part follows from the well known formula

$$
e^{-l_{i}} \cdot X_{i} \cdot e^{l_{i}}=X_{i}+\sum_{m=1}^{\infty} \frac{1}{m !}\left[\left[X_{i}, l_{i}\right] l_{i}^{m-1}\right] .
$$

The second part is obvious, so it rests to show the last statement of the lemma. It is well known that the Lie algebra of the group of automorphisms of a $\mathbf{Q}_{l}$-algebra is the Lie algebra of derivations of this $\mathbf{Q}_{l}$-algebra. Let $D$ be a derivation of the $\mathbf{Q}_{l}$-algebra $\mathbf{Q}_{l}\{\{\mathbf{X}\}\}$. Suppose that $\exp t D \in$ Aut $^{*}\left(\mathbf{Q}_{l}\{\{\mathbf{X}\}\}\right)$. Then $(\exp t D)\left(X_{i}\right)=e^{-l_{i}(t)} \cdot X_{i} \cdot e^{l_{i}(t)}$ for $i=1, \ldots, n$. The elements $l_{i}(t)$ are in $L(\mathbf{X})$. We can suppose that the coefficient of $l_{i}(t)$ at $X_{i}$ vanishes. Then we have $l_{i}(0)=0$ and $l_{i}(t)$ depends smoothly on $t$. Hence $A_{i}:=\lim _{t \rightarrow 0} \frac{1}{t} l_{i}(t)$ exists and belongs to $L(\mathbf{X})$. Comparing Taylor developments of $(\exp t D)\left(X_{i}\right)$ and $e^{-l_{i}(t)} \cdot X_{i} \cdot e^{l_{i}(t)}$ we get $D\left(X_{i}\right)=\left[X_{i}, A_{i}\right]$ for $i=1, \ldots, n$. Therefore $D$ belongs to $\operatorname{Der}^{*}\left(\mathbf{Q}_{l}\{\{\mathbf{X}\}\}\right)$.

Proposition 5.1.3. Let $\sigma \in G_{K}$. Then $\varphi_{x}(\sigma) \in \operatorname{Aut}^{*}\left(\mathbf{Q}_{l}\{\{\mathbf{X}\}\}\right)$ and $\log \varphi_{x}(\sigma) \in \operatorname{Der}^{*}\left(\mathbf{Q}_{l}\{\{\mathbf{X}\}\}\right)$.

Proof. It follows from Proposition 2.2.1 that

$$
\sigma_{x}\left(X_{i}\right)=\left(\Lambda_{\left(\gamma_{i}, x\right)}(\sigma)\right)^{-1} \cdot \chi(\sigma) X_{i} \cdot \Lambda_{\left(\gamma_{i}, x\right)}(\sigma)
$$

for $i=1, \ldots, n$. Hence $\varphi_{x}(\sigma) \in \operatorname{Aut}^{*}\left(\mathbf{Q}_{l}\{\{\mathbf{X}\}\}\right)$. It follows from Lemma 5.1.1 that $\log \varphi_{x}(\sigma) \in \operatorname{Der}^{*}\left(\mathbf{Q}_{l}\{\{\mathbf{X}\}\}\right)$.

To give an explicit formula for $\log \varphi_{x}\left(X_{i}\right)$ we need to study Galois actions on torsors of paths. The action of Galois groups on torsors of paths requires to introduce semi-direct products of Lie algebras. Below we give the necessary definitions.

Let $L$ be a Lie algebra and let $\mathcal{D}$ be a Lie subalgebra of the algebra of Lie derivations of $L$. We equip the direct product $L \times \mathcal{D}$ with a Lie bracket

$$
\left[(l, D),\left(l_{1}, D_{1}\right)\right]:=\left(\left[l, l_{1}\right]+D\left(l_{1}\right)-D_{1}(l),\left[D, D_{1}\right]\right) .
$$

The resulting Lie algebra we denote by $L \tilde{\times} \mathcal{D}$ and we call it a semi-direct product of $L$ and $\mathcal{D}$.

If $g \in \mathbf{Q}_{l}\{\{\mathbf{X}\}\}$ then $L_{g}$ denotes left multiplication by $g . L_{\exp (L(\mathbf{X}))}$ is the set of left multiplications by elements of $\exp (L(\mathbf{X}))$ and $L_{L(\mathbf{X})}$ is the set of left multiplications by elements of $L(\mathbf{X})$. 
LEMMA 5.1.4. Let $\mathcal{G}$ be a subgroup of $\mathrm{GL}\left(\mathbf{Q}_{l}\{\{\mathbf{X}\}\}\right)$ generated by $L_{\exp (L(\mathbf{X}))}$ and $\mathrm{Aut}^{*}\left(\mathbf{Q}_{l}\{\{\mathbf{X}\}\}\right)$. Then $\mathcal{G}$ is a semi-direct product of $L_{\exp (L(\mathbf{X}))}$ and $\mathrm{Aut}^{*}\left(\mathbf{Q}_{l}\{\{\mathbf{X}\}\}\right)$, which we denote by $L_{\exp (L(\mathbf{X}))} \tilde{\times}$ Aut $^{*}\left(\mathbf{Q}_{l}\{\{\mathbf{X}\}\}\right)$. The Lie algebra of $L_{\exp (L(\mathbf{X}))} \tilde{\times} \operatorname{Aut}^{*}\left(\mathbf{Q}_{l}\{\{\mathbf{X}\}\}\right)$ is equal to a semi-direct product of Lie algebras $L_{L(\mathbf{X})} \tilde{\times} \operatorname{Der}^{*} L(\mathbf{X}) \approx L(\mathbf{X}) \tilde{\times} \operatorname{Der}^{*} L(\mathbf{X})$.

Proof. Let $f, f_{1} \in \exp (L(\mathbf{X}))$ and $\phi, \phi_{1} \in \operatorname{Aut}^{*}\left(\mathbf{Q}_{l}\{\{\mathbf{X}\}\}\right)$. Then we have

$$
\left(L_{f} \circ \phi\right) \circ\left(L_{f_{1}} \circ \phi_{1}\right)=L_{f \cdot \phi\left(f_{1}\right)} \circ\left(\phi \circ \phi_{1}\right) .
$$

This implies that $\mathcal{G}$ is a semi-direct product of $L_{\exp (L(\mathbf{X}))}$ and Aut* $\left(\mathbf{Q}_{l}\{\{\mathbf{X}\}\}\right)$. It follows from Lemma 5.1.1 that the Lie algebra of Aut* $\left(\mathbf{Q}_{l}\{\{\mathbf{X}\}\}\right)$ is $\operatorname{Der}^{*} L(\mathbf{X})$. The Lie algebra of $L_{\exp (L(\mathbf{X}))}$ is $L_{L(\mathbf{X})}$. Hence the Lie algebra of $\mathcal{G}$ is equal to $L_{L(\mathbf{X})} \times \operatorname{Der}^{*} L(\mathbf{X})$ as a vector space.

Let $f, g \in L(\mathbf{X})$ and let $D, E \in \operatorname{Der}^{*} L(\mathbf{X})$. Observe that $L_{f}+D$ is the tangent vector at $t=1$ to the curve $t \rightarrow L_{\exp t f} \circ \exp t D$. To calculate a Lie bracket of the Lie algebra of $\mathcal{G}$ we need to calculate the coefficient at $t^{2}$ of the commutator

$$
\left(L_{\exp t f} \circ \exp t D, L_{\exp t g} \circ \exp t E\right)
$$

This coefficient is equal $L_{[f, g]+D(g)-E(f)}+[D, E]$. This shows that the Lie algebra of $\mathcal{G}$ is the semi-direct product of Lie algebras $L_{L(\mathbf{X})} \tilde{\times} \operatorname{Der}^{*} L(\mathbf{X}) \approx$ $L(\mathbf{X}) \tilde{\times} \operatorname{Der}^{*} L(\mathbf{X})$.

Proposition 5.1.5. Let $\sigma \in G_{K}$. Then $\psi_{x, p}(\sigma) \in L_{\exp (L(\mathbf{X}))} \tilde{\times}$ Aut $^{*}$ $\left(\mathbf{Q}_{l}\{\{\mathbf{X}\}\}\right)$ and $\log \psi_{x, p}(\sigma) \in L_{L(\mathbf{X})} \tilde{\times} \operatorname{Der}^{*} L(\mathbf{X})$.

Proof. Let $\sigma \in G_{K}$ and $w \in \mathbf{Q}_{l}\{\{\mathbf{X}\}\}$. We have

$$
\psi_{x, p}(\sigma)(w)=\Lambda_{(p, x)}(\sigma) \cdot \varphi_{x}(\sigma)(w)
$$

It follows from (5.1.6) that $\psi_{x, p}(\sigma)$ belongs to the semi-direct product

$$
L_{\exp (L(\mathbf{X}))} \tilde{x} \operatorname{Aut}^{*}\left(\mathbf{Q}_{l}\{\{\mathbf{X}\}\}\right) .
$$

The Lie algebra of the semi-direct product of groups $L_{\exp (L(\mathbf{X}))} \tilde{\times}$ Aut* $\left(\mathbf{Q}_{l}\{\{\mathbf{X}\}\}\right)$ is equal to a semi-direct product of Lie algebras $L_{L(\mathbf{X})} \tilde{x}$ $\operatorname{Der}^{*} L(\mathbf{X}) \approx L(\mathbf{X}) \tilde{\times} \operatorname{Der}^{*} L(\mathbf{X})$ by Lemma 5.1.4. Therefore $\log \psi_{x, p}(\sigma) \in$ $L_{L(\mathbf{X})} \tilde{x} \operatorname{Der}^{*} L(\mathbf{X})$. This finishes the proof of the proposition. 
Below we shall calculate both components of $\log \psi_{x, p}(\sigma)$.

We denote by $\bigcirc$ a product given by the Baker-Campbell-Hausdorff formula (BCH formula) (see [MKS, Theorem 5.19]).

Proposition 5.1.7. The element $\log \psi_{x, p}(\sigma)(1) \in L(\mathbf{X})$ and we have

$$
\log \psi_{x, p}(\sigma)=L_{\left(\log \psi_{x, p}(\sigma)\right)(1)}+\log \varphi_{x}(\sigma)
$$

Proof. Let $g, h \in L(\mathbf{X})$ and $D \in \operatorname{Der}^{*}\left(\mathbf{Q}_{l}\{\{\mathbf{X}\}\}\right)$. Then $\left[L_{g}, L_{h}\right]=$ $L_{[g, h]}$ and $\left[D, L_{g}\right]=L_{D(g)}$. Hence all terms of $\log \psi_{x, p}(\sigma)-\log \varphi_{x}(\sigma)=$

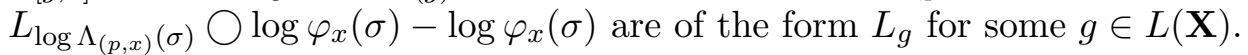
Therefore $\log \psi_{x, p}(\sigma)=L_{g}+\log \varphi_{x}(\sigma)$ for some $g \in L(\mathbf{X})$. Evaluating both sides of the equality at 1 we get that $g=\left(\psi_{x, p}(\sigma)\right)(1)$. This finishes the proof of the proposition.

Proposition 5.1.8. Let $\sigma \in G_{K}$. Then we have

$$
\log \varphi_{x}(\sigma)\left(X_{k}\right)=\left[X_{k} ;\left(\log \psi_{x, \gamma_{k}}(\sigma)\right)(1)\right]
$$

for $k=1, \ldots, n$.

Proof. One computes easily that

$$
\begin{aligned}
& \left(\log \psi_{x, \gamma_{k}}(\sigma)\right)(1)=\left(\Lambda_{\left(\gamma_{k}, x\right)}(\sigma)-1\right) \\
& \quad-\frac{1}{2}\left(\Lambda_{\left(\gamma_{k}, x\right)}(\sigma) \cdot \varphi_{x}(\sigma)\left(\Lambda_{\left(\gamma_{k}, x\right)}(\sigma)\right)-2 \Lambda_{\left(\gamma_{k}, x\right)}(\sigma)+1\right) \\
& +\frac{1}{3}\left(\Lambda_{\left(\gamma_{k}, x\right)}(\sigma) \cdot \varphi_{x}(\sigma)\left(\Lambda_{\left(\gamma_{k}, x\right)}(\sigma)\right) \cdot \varphi_{x}(\sigma)^{2}\left(\Lambda_{\left(\gamma_{k}, x\right)}(\sigma)\right)\right. \\
& \left.\quad-3 \Lambda_{\left(\gamma_{k}, x\right)}(\sigma) \cdot \varphi_{x}(\sigma)\left(\Lambda_{\left(\gamma_{k}, x\right)}(\sigma)\right)+3 \Lambda_{\left(\gamma_{k}, x\right)}(\sigma)-1\right) \cdots
\end{aligned}
$$

This implies that $\left(\log \psi_{x, \gamma_{k}}(\sigma)\right)\left(X_{k}\right)=X_{k} \cdot\left(\left(\log \psi_{x, \gamma_{k}}(\sigma)\right)(1)\right)$. Now it follows from Proposition 5.1.7 that $\log \varphi_{x}(\sigma)\left(X_{k}\right)=\left[X_{k} ;\left(\log \psi_{x, \gamma_{k}}(\sigma)\right)(1)\right]$.

5.2. The main object of our study are coefficients of the operator $\log \psi_{x, p}(\sigma)$ for varing $\sigma$ (see Definition 5.3.0). The element $\log \psi_{x, p}(\sigma) \in$ $L_{L(\mathbf{X})} \tilde{\tilde{\times}} \operatorname{Der}^{*} L(\mathbf{X})$. Hence to study these coefficients we need to study linear forms on the Lie algebra $L_{L(\mathbf{X})} \tilde{\times} \operatorname{Der}^{*} L(\mathbf{X})$ and on various Lie subalgebras of this Lie algebra. First we define suitable linear forms which evaluated on the element $\log \psi_{x, p}(\sigma)$ gives coefficients. Next we are studying properties of the operators induced by the Lie brackets on these linear forms.

The free Lie algebra Lie $(\mathbf{X})$ (resp. the completed free Lie algebra $L(\mathbf{X})$ ) has an obvious $\mathbf{Q}$-structure - a free Lie algebra over $\mathbf{Q}$ on the set $\mathbf{X}$ (resp. a 
completed free Lie algebra over $\mathbf{Q}$ on the set $\mathbf{X})$. Therefore the Lie algebras of derivations $\operatorname{Der} \operatorname{Lie}(\mathbf{X})$ and $\operatorname{Der}{ }^{*} \operatorname{Lie}(\mathbf{X})$ (resp. Der $L(\mathbf{X})$ and $\operatorname{Der}^{*} L(\mathbf{X})$ ) have also $\mathbf{Q}$-structures.

Let $\operatorname{Lie}(\mathbf{X})_{m}$ be a vector subspace of Lie(X) of homogenous elements of degree $m$. Let $\left(\operatorname{Lie}(\mathbf{X})_{m}\right)^{*}$ be the dual vector space of the finite dimentional vector space $\operatorname{Lie}(\mathbf{X})_{m}$. We define the graded dual Lie $(\mathbf{X})^{\diamond}$ of the free Lie algebra by

$$
\operatorname{Lie}(\mathbf{X})^{\diamond}:=\bigoplus_{m=1}^{\infty}\left(\operatorname{Lie}(\mathbf{X})_{m}\right)^{*}
$$

Let $\left\langle X_{i}\right\rangle$ be a vector subspace of $\operatorname{Lie}(\mathbf{X})$ generated by $X_{i}$ and let $\left\langle X_{i}\right\rangle^{*}$ be the dual vector space. We define the subspace of $\operatorname{Lie}(\mathbf{X})^{\diamond}$ of linear forms killing $\left\langle X_{i}\right\rangle$ by

$$
\left(\operatorname{Lie}(\mathbf{X}) /\left\langle X_{i}\right\rangle\right)^{\diamond}:=\operatorname{ker}\left(\operatorname{Lie}(\mathbf{X})^{\diamond} \rightarrow\left\langle X_{i}\right\rangle^{*}\right)
$$

We shall define the graded dual of the semi-direct product $\operatorname{Lie}(\mathbf{X}) \tilde{\times} \operatorname{Der}^{*}$ $\operatorname{Lie}(\mathbf{X})$. We start with the following observation. Let $D \in \operatorname{Der}{ }^{*} \operatorname{Lie}(\mathbf{X})$ be such that $D\left(X_{i}\right)=\left[X_{i}, A_{i}\right]$ for $i=1, \ldots, n$. The map

$$
f: \operatorname{Der}^{*} \operatorname{Lie}(\mathbf{X}) \longrightarrow \bigoplus_{i=1}^{n}\left(\operatorname{Lie}(\mathbf{X}) /\left\langle X_{i}\right\rangle\right)
$$

given by $f(D)=\left(A_{1}, \ldots, A_{n}\right)$ is an isomorphism of vector spaces. The isomorphism $f$ is compatible with $\mathbf{Q}$-structures on both vector spaces. The isomorphism $f$ identifies $\operatorname{Der}{ }^{*} \operatorname{Lie}(\mathbf{X})$ with $\bigoplus_{i=1}^{n}\left(\operatorname{Lie}(\mathbf{X}) /\left\langle X_{i}\right\rangle\right)$. We define the graded dual of the Lie algebra $\operatorname{Der}{ }^{*} \operatorname{Lie}(\mathbf{X})$ by

$$
\left(\operatorname{Der}^{*} \operatorname{Lie}(\mathbf{X})\right)^{\diamond}:=\bigoplus_{i=1}^{n}\left(\operatorname{Lie}(\mathbf{X}) /\left\langle X_{i}\right\rangle\right)^{\diamond}
$$

The dual of a semi-direct product of two Lie algebras is a direct sum of duals of these two Lie algebras. Hence we set

$$
\left(\operatorname{Lie}(\mathbf{X}) \tilde{\times} \operatorname{Der}^{*} \operatorname{Lie}(\mathbf{X})\right)^{\diamond}:=\operatorname{Lie}(\mathbf{X})^{\diamond} \oplus\left(\operatorname{Der}^{*} \operatorname{Lie}(\mathbf{X})\right)^{\diamond}
$$

Definition 5.2.0. Let $V$ be a vector space. We say that $V$ is a Lie coalgebra if $V$ is equipped with a linear map $d: V \rightarrow V \otimes V$ satisfying

i) $\tau \circ d+d=0$, where $\tau(a \otimes b)=b \otimes a$; 
ii) $\sum_{i=0}^{2} \sigma^{i} \circ\left(d \otimes i d_{V}\right) \circ d=0$, where $\sigma(a \otimes b \otimes c)=b \otimes c \otimes a$.

It follows from i) that $d$ factors through $d: V \rightarrow V \wedge V$, where

$$
V \wedge V=\left\{\sum_{i \in I} n_{i}\left(a_{i} \otimes b_{i}-b_{i} \otimes a_{i}\right) \in V \otimes V\right\}
$$

Farther we shall also denote $V \wedge V$ by $\wedge^{2} V$.

LEMMA 5.2.1. $\quad$ i) If $V$ is a Lie coalgebra then the dual vector space $V^{*}$ equipped with [ ] $:=d^{*}: V^{*} \otimes V^{*} \rightarrow V^{*}$ is a Lie algebra.

ii) If $L$ is a Lie algebra then $L^{*}$ equipped with $d:=[]^{*}: L^{*} \rightarrow L^{*} \otimes L^{*}$ is a Lie coalgebra.

Corollary 5.2.2. The vector spaces $\operatorname{Lie}(\mathbf{X})^{\diamond}$, $\left(\operatorname{Der}^{*} \operatorname{Lie}(\mathbf{X})\right)^{\diamond}$ and $\left(\operatorname{Lie}(\mathbf{X}) \tilde{\times} \operatorname{Der}^{*} \operatorname{Lie}(\mathbf{X})\right)^{\diamond}$ equipped with $d:=[]^{*}$ are Lie coalgebras.

Proof. The dual vector spaces $\operatorname{Lie}(\mathbf{X})^{*},\left(\operatorname{Der}{ }^{*} \operatorname{Lie}(\mathbf{X})\right)^{*}$ and $(\operatorname{Lie}(\mathbf{X}) \tilde{x}$ Der* Lie $(\mathbf{X}))^{*}$ equipped with $d:=[]^{*}$ are Lie coalgebras. Observe that $d$ preserves $\operatorname{Lie}(\mathbf{X})^{\diamond},\left(\operatorname{Der}^{*} \operatorname{Lie}(\mathbf{X})\right)^{\diamond}$ and $\left(\operatorname{Lie}(\mathbf{X}) \tilde{\times} \operatorname{Der}^{*} \operatorname{Lie}(\mathbf{X})\right)^{\diamond}$. Hence these vector spaces are also Lie coalgebras.

5.2.3. The vector spaces $\operatorname{Lie}(\mathbf{X})^{\diamond},\left(\operatorname{Der}{ }^{*} \operatorname{Lie}(\mathbf{X})\right)^{\diamond}$ and $\left(\operatorname{Lie}(\mathbf{X}) \tilde{\times} \operatorname{Der}^{*}\right.$ $\operatorname{Lie}(\mathbf{X}))^{\diamond}$ are canonically embedded as Lie coalgebras into $L(\mathbf{X})^{*}$, (Der* $L(\mathbf{X}))^{*}$ and $\left(L(\mathbf{X}) \tilde{\times} \operatorname{Der}^{*} L(\mathbf{X})\right)^{*}$ respectively. When we view these vector spaces as vector subspaces of $L(\mathbf{X})^{*},\left(\operatorname{Der}^{*} L(\mathbf{X})\right)^{*}$ and $\left(L(\mathbf{X}) \tilde{\times} \operatorname{Der}^{*} L(\mathbf{X})\right)^{*}$ then we denote them by $L(\mathbf{X})^{\diamond},\left(\operatorname{Der}^{*} L(\mathbf{X})\right)^{\diamond}$ and $\left(L(\mathbf{X}) \tilde{\times} \operatorname{Der}^{*} L(\mathbf{X})\right)^{\diamond}$ respectively.

5.3. Below we shall give the very definition of the $l$-adic iterated integrals. Observe that the element $\left(\log \psi_{x, p}(\sigma)\right)(1)$ is a Lie element in $\mathbf{Q}_{l}\{\{\mathbf{X}\}\}$ by Proposition 5.1.7.

Definition 5.3.0. Let us fix a Hall base $\mathcal{B}$ of $\operatorname{Lie}(\mathbf{X})$. Let $\sigma \in G_{K}$. We set

$$
a_{x, p}(\sigma):=\left(\log \psi_{x, p}(\sigma)\right)(1)=\sum_{e \in \mathcal{B}} a_{x, p}^{e}(\sigma) \cdot e .
$$

Let $\phi \in L(\mathbf{X})^{\diamond}$ be a linear form defined over $\mathbf{Q}$. We set

$$
a_{x, p}^{\phi}(\sigma):=\phi\left(\left(\log \psi_{x, p}(\sigma)\right)(1)\right)
$$


The functions $a_{x, p}^{e}: G_{K} \rightarrow \mathbf{Q}_{l}$ we shall call $l$-adic iterated integrals.

If $e \in \mathcal{B}$ then we denote by $e^{*}$ the dual vector with respect to this base $\mathcal{B}$. Observe that the set $\left\{e^{*}\right\}_{e \in \mathcal{B}}$ is a linear base of $\operatorname{Lie}(\mathbf{X})^{\diamond}$. Hence any $a_{x, p}^{\phi}$ is a linear combination of a finite number of $a_{x, p}^{e}$.

TheOREm 5.3.1. Let $e \in \mathcal{B}$ be an element of degree $i$. We have:

i) $a_{x, p}^{e}(\sigma)=0$ for $\sigma \in H_{i+1}$.

ii) $a_{x, p}^{e}(\tau \cdot \sigma)=a_{x, p}^{e}(\tau)+a_{x, p}^{e}(\sigma)$ for any $\tau, \sigma \in H_{i}$.

iii) The homomorphism $a_{x, p \mid H_{i}}^{e}: H_{i} \rightarrow \mathbf{Q}_{l}(i)$ is compatible with the action of $\operatorname{Gal}\left(K\left(\mu_{l^{\infty}}\right) / K\right)$ on $H_{i}$ and $\mathbf{Q}_{l}(i)$.

iv) The homomorphism $a_{x, p \mid H_{i}}^{e}: H_{i} \rightarrow \mathbf{Q}_{l}(i)$ depends only on $z$ and $v$. It does not depend on the choice of geometric generators $x$ (in a given permutation class) and on a choice of a path $p$ from $v$ to $z$.

Proof. The point i) follows from the definition of the group $H_{i}$ and from Lemma 4.1.1. Let $\tau, \sigma \in H_{i}$. Then $\psi_{x, p}(\sigma)=\sigma_{x, p}, \psi_{x, p}(\tau)=\tau_{x, p}$ and $\psi_{x, p}(\tau \cdot \sigma)=(\tau \cdot \sigma)_{x, p}$ It follows from the point i) that

$$
\left(\log \sigma_{x, p}\right)(1)=\sum_{e \in \mathcal{B}^{i}} a_{x, p}^{e}(\sigma) \cdot e+\sum_{j \geq i+1} \sum_{e \in \mathcal{B}^{j}} a_{x, p}^{e}(\sigma) \cdot e .
$$

We have $(\tau \cdot \sigma)_{x, p}=\tau_{x, p} \circ \sigma_{x, p}$. The BCH formula implies

$$
\log (\tau \cdot \sigma)_{x, p}=\log \tau_{x, p}+\log \sigma_{x, p}+\frac{1}{2}\left[\log \tau_{x, p}, \log \sigma_{x, p}\right]+\cdots .
$$

Evaluating both sides of the equality at 1 we get

$$
\left(\log (\tau \cdot \sigma)_{x, p}\right)(1)=\left(\log \tau_{x, p}\right)(1)+\left(\log \sigma_{x, p}\right)(1)+A(\tau, \sigma)(1),
$$

where $A(\tau, \sigma)=\frac{1}{2}\left[\log \tau_{x, p}, \log \sigma_{x, p}\right]+\cdots$. It follows from the point i) that terms of degree $i$ of $A(\tau, \sigma)(1)$ vanish. Hence $a_{x, p}^{e}(\tau \cdot \sigma)=a_{x, p}^{e}(\tau)+a_{x, p}^{e}(\sigma)$ for $e \in \mathcal{B}^{i}$ and $\tau, \sigma \in H_{i}$. The points iii) and iv) follow from Lemma 3.2.1.

We recall from Proposition 5.1.7 that

$$
\log \psi_{x, p}(\sigma)=L_{\left(\log \psi_{x, p}(\sigma)\right)(1)}+\log \varphi_{x}(\sigma) .
$$

The $l$-adic iterated integrals introduced in Definition 5.3.0 are coefficients of the element $\left(\log \psi_{x, p}(\sigma)\right)(1)$. We must also study coefficients of the operator $\log \varphi_{x}(\sigma)$. We recall that $\varphi_{x}(\sigma)$ is an automorphism of $\mathbf{Q}_{l}\{\{\mathbf{X}\}\}$ induced by the action of $\sigma$ on $\pi_{1}\left(X_{\bar{K}} ; v\right)$ twisted by the cyclotomic character (see Section 4). Hence the operator $\varphi_{x}(\sigma)$ depends only on a choice of geometric generators $x=\left(x_{1}, \ldots, x_{n+1}\right)$ and on a choice of a base point $v$. 
Definition 5.3.3. Let $\varepsilon \in\left(\operatorname{Der}^{*} L(\mathbf{X})\right)^{\diamond}$ be a linear form of degree $m$ and let $\sigma \in G_{K}$. We set

$$
\varepsilon(v)(\sigma):=\varepsilon\left(\log \varphi_{x}(\sigma)\right)
$$

Observe that $\varepsilon(v)$ is a function from $G_{K}$ to $\mathbf{Q}_{l}$. We shall use functions $\varepsilon(v)$ to express the action of the operator $d$ on $l$-adic iterated integrals. Any function $\varepsilon(v)$ is in fact a linear combination of $l$-adic iterated integrals defined in Definition 5.3.0. However it is still very useful to have a separated notation for these functions.

Proposition 5.3.4. There are $e_{1}, \ldots, e_{r} \in \mathcal{B}_{m}$ and $\alpha_{k, i} \in \mathbf{Q}_{l}$ for $0<$ $k<n+1$ and $0<i<r+1$ such that

$$
\varepsilon(v)=\sum_{k=1}^{n} \sum_{i=1}^{r} \alpha_{k, i} a_{x, \gamma_{k}}^{e_{i}}
$$

If $\varepsilon$ is defined over $\mathbf{Q}$ then $\alpha_{k, i}$ are in $\mathbf{Q}$.

Proof. The proposition follows from Proposition 5.1.8.

We shall see later that the function $a_{x, p}^{e}: G_{K} \rightarrow \mathbf{Q}_{l}$ depends on a choice of a path $p$ from $v$ to $z$. Assume that $e$ is of degree $m$. It follows from Theorem 5.3.1 iv) that the restriction of $a_{x, p}^{e}$ to the subgroup $H_{m}(X ; z, v)$ depends only on $z$ and $v$. It does not depend on a choice of a path $p$. This motivate the following definition.

Definition 5.3.5. Let $e \in \mathcal{B}$ be an element of degree $m$ and let $\varphi \in$ $L(\mathbf{X})^{\diamond}$ be a linear form of degree $m$. We set

$$
\mathcal{L}^{e}(z, v):=a_{x, p \mid H_{m}(X ; z, v)}^{e} \quad \text { and } \quad \mathcal{L}^{\varphi}(z, v):=a_{x, p \mid H_{m}(X ; z, v)}^{\varphi} .
$$

Let $\varepsilon \in\left(\operatorname{Der}^{*} L(\mathbf{X})\right)^{\diamond}$ be a linear form of degree $m$. We set

$$
\mathcal{L}^{\varepsilon}(v):=\varepsilon(v)_{\mid H_{m}(X ; z, v)} \cdot
$$

It follows from Proposition 5.3.4 that

$$
\mathcal{L}^{\varepsilon}(v)=\sum_{k=1}^{n} \sum_{i=1}^{r} \alpha_{k, i} \mathcal{L}^{e_{i}}\left(v_{k}, v\right) .
$$




\section{$\S 6$. Cocycle conditions}

6.0. It follows from Proposition 1.0.7 that the function $\mathfrak{f}_{p}: G_{K} \rightarrow$ $\pi_{1}\left(X_{\bar{K}} ; v\right)$ is a cocycle. Similarly Lemma 4.2.1 implies that the functions $\varphi_{x}: G_{K} \rightarrow \operatorname{Aut}\left(\mathbf{Q}_{l}\{\{\mathbf{X}\}\}\right)$ and $\psi_{x, p}: G_{K} \rightarrow \operatorname{GL}\left(\mathbf{Q}_{l}\{\{\mathbf{X}\}\}\right)$ are cocycles. The map ()$_{x, p}: G_{K\left(\mu_{l} \infty\right)} \rightarrow \mathrm{GL}\left(\mathbf{Q}_{l}\{\{\mathbf{X}\}\}\right)$ is a homomorphism. However coefficients of these matrix valued functions usually are not cocycles or homomorphisms.

Let $\varphi \in L(\mathbf{X})^{\diamond}$ be a linear form of degree $m$. The function $a_{x, p}^{\varphi}$ : $G_{K\left(\mu_{l} \infty\right)} \rightarrow \mathbf{Q}_{l}(m)$ (resp. $a_{x, p}^{\varphi}: G_{K} \rightarrow \mathbf{Q}_{l}(m)$ ) usually is not a homomorphism (resp. a cocycle). We are looking for conditions when a linear combination of various $a_{x, p}^{\varphi}$ with $\mathbf{Q}_{l}$ coefficients is a homomorphism (resp. a cocycle).

Let $T$ be a finite subset of $\hat{X}(K)^{2}$ containing a pair $(z, v)$. It follows from Section 5.0 that $a_{x, p}^{\varphi}$ and $\varepsilon(v)$ can be also considered as functions from the Lie algebra $\operatorname{Lie}\left(\mathcal{K}_{1}^{T}(X) / \mathcal{K}_{\infty}^{T}(X)\right)$ to $\mathbf{Q}_{l}$.

Lemma 6.0.1. Let $\varphi \in L(\mathbf{X})^{\diamond}$ be a linear form of degree $m$ and let $T$ be a finite subset of $\hat{X}(K)^{2}$ containing a pair $(z, v)$. Assume that

$$
d(\varphi)=\sum_{k+j=m}\left(\sum_{e \in \mathcal{B}_{k}, e^{\prime} \in \mathcal{B}_{j}} c_{e, e^{\prime}} e^{*} \wedge e^{\prime *}+\sum_{e \in \mathcal{B}_{k}, \varepsilon \in\left(\operatorname{Der}^{*} L(\mathbf{X})\right)^{j}} b_{e, \varepsilon} e^{*} \wedge \varepsilon\right)
$$

in $\bigwedge^{2}\left(L(\mathbf{X}) \tilde{x} \operatorname{Der}^{*} L(\mathbf{X})\right)^{\diamond}$. Then we have

$d\left(a_{x, p}^{\varphi}\right)=\sum_{k+j=m}\left(\sum_{e \in \mathcal{B}_{k}, e^{\prime} \in \mathcal{B}_{j}} c_{e, e^{\prime}} a_{x, p}^{e} \wedge a_{x, p}^{e^{\prime}}+\sum_{e \in \mathcal{B}_{k}, \varepsilon \in\left(\operatorname{Der}^{*} L(\mathbf{X})\right)^{j}} b_{e, \varepsilon} a_{x, p}^{e} \wedge \varepsilon(v)\right)$ in $\bigwedge^{2}\left(\operatorname{Lie}\left(\mathcal{K}_{1}^{T}(X) / \mathcal{K}_{\infty}^{T}(X)\right)\right)^{*}$, where

$$
\left(\operatorname{Lie}\left(\mathcal{K}_{1}^{T}(X) / \mathcal{K}_{\infty}^{T}(X)\right)\right)^{*}:=\operatorname{Hom}_{\mathbf{Z}_{l}}\left(\operatorname{Lie}\left(\mathcal{K}_{1}^{T}(X) / \mathcal{K}_{\infty}^{T}(X)\right) ; \mathbf{Q}_{l}\right) .
$$

Proof. The lemma is an obvious consequence of the fact that the map

$$
\operatorname{Lie}()_{x, p}: \operatorname{Lie}\left(\mathcal{K}_{1}^{T}(X) / \mathcal{K}_{\infty}^{T}(X)\right) \longrightarrow L_{L(\mathbf{X})} \tilde{\times} \operatorname{Der}^{*} L(\mathbf{X})
$$

is a morphism of Lie algebras.

Proposition 6.0.2. Let $\left(z_{i}, v_{i}\right) \in \hat{X}(K)^{2}$, let $\varphi_{i} \in L(\mathbf{X})^{\diamond}$ be a linear form of degree $m$, let $p_{i}$ be a path from $v_{i}$ to $z_{i}$ and let $x_{i}$ be a sequence of geometric generators of $\pi_{1}\left(X(\mathbf{C}) ; v_{i}\right)$ for $i=1, \ldots, N$. Let $n_{1}, \ldots, n_{N}$ be in $\mathbf{Q}_{l}$. Let $T$ be a finite subset of $\hat{X}(K)^{2}$ containing pairs $\left(z_{i}, v_{i}\right)$ for $i=1, \ldots, N$. 
i) Assume that $d\left(\sum_{i=1}^{N} n_{i} a_{x_{i}, p_{i}}^{\varphi_{i}}\right)=0$ in $\bigwedge^{2}\left(\operatorname{Lie}\left(\mathcal{K}_{1}^{T}(X) / \mathcal{K}_{\infty}^{T}(X)\right)\right)^{*}$. Then $\sum_{i=1}^{N} n_{i} a_{x_{i}, p_{i}}^{\varphi_{i}}$ is a homomorphism from $\mathcal{K}_{1}^{T}(X) / \mathcal{K}_{\infty}^{T}(X)$ to $\mathbf{Q}_{l}(m)$.

ii) Assume that for any $\tau$ and $\sigma$ in $G_{K}$

$\sum_{i=1}^{N} n_{i} \varphi_{i}\left(\left[\cdots\left[\cdots\left[\log \psi_{x_{i}, p_{i}}(\tau), \log \psi_{x_{i}, p_{i}}(\sigma)^{\chi(\tau)^{-1}}\right], \log \psi_{x_{i}, p_{i}}(\tau)\right] \cdots\right](1)\right)=0$

for all Lie brackets of lengths $2,3, \ldots, m$. Then $\sum_{i=1}^{N} n_{i} a_{x_{i}, p_{i}}^{\varphi_{i}}$ is a cocycle on $G_{K}$ with values in $\mathbf{Q}_{l}(m)$.

Proof. We start with the proof of the first part of the proposition. Let $\tau, \sigma \in \mathcal{K}_{1}^{T}(X)$. Let us set $T_{i}=\log \tau_{x_{i}, p_{i}}$ and $S_{i}=\log \sigma_{x_{i}, p_{i}}$. The equality $\tau_{x_{i}, p_{i}} \circ \sigma_{x_{i}, p_{i}}=(\tau \sigma)_{x_{i}, p_{i}}$ implies

$$
\log (\tau \sigma)_{x_{i}, p_{i}}=T_{i}+S_{i}+\frac{1}{2}\left[T_{i}, S_{i}\right]-\frac{1}{12}\left[\left[T_{i}, S_{i}\right] T_{i}\right]+\cdots .
$$

Evaluating $\varphi_{i}$ on the last equality we get

$$
a_{x_{i}, p_{i}}^{\varphi_{i}}(\tau \sigma)=a_{x_{i}, p_{i}}^{\varphi_{i}}(\tau)+a_{x_{i}, p_{i}}^{\varphi_{i}}(\sigma)+\frac{1}{2} \varphi_{i}\left(\left[T_{i}, S_{i}\right]\right)-\frac{1}{12} \varphi_{i}\left(\left[\left[T_{i}, S_{i}\right] T_{i}\right]\right)+\cdots .
$$

Observe that $\varphi_{i}\left(\left[T_{i}, S_{i}\right]\right)=d \varphi_{i}\left(T_{i} \otimes S_{i}\right)=d a_{x_{i}, p_{i}}^{\varphi_{i}}(\log \tau \otimes \log \sigma)$. Hence $\sum_{i=1}^{N} n_{i} \varphi_{i}\left(\left[T_{i}, S_{i}\right]\right)=0$. Observe that $\varphi([[T, S] R])=((d \otimes i d) \circ d)(\varphi)(T \otimes S \otimes$ $R)$. This implies $\sum_{i=1}^{N} n_{i} \varphi_{i}\left(\left[\left[T_{i}, S_{i}\right] T_{i}\right]\right)=0$. We apply the same arguments to others brackets and finally we get

$$
\sum_{i=1}^{N} n_{i} a_{x_{i}, p_{i}}^{\varphi_{i}}(\tau \sigma)=\sum_{i=1}^{N} n_{i} a_{x_{i}, p_{i}}^{\varphi_{i}}(\tau)+\sum_{i=1}^{N} n_{i} a_{x_{i}, p_{i}}^{\varphi_{i}}(\sigma)
$$

Now we assume that $\tau, \sigma \in G_{K}$. The equality

$$
\psi_{x_{i}, p_{i}}(\tau \sigma)=\psi_{x_{i}, p_{i}}(\tau) \circ \psi_{x_{i}, p_{i}}(\sigma)^{\chi(\tau)^{-1}}
$$

(see Lemma 4.2.1) implies that

$$
\begin{aligned}
\log \psi_{x_{i}, p_{i}}(\tau \sigma)= & \log \psi_{x_{i}, p_{i}}(\tau) \bigcirc \log \psi_{x_{i}, p_{i}}(\sigma)^{\chi(\tau)^{-1}} \\
= & \log \psi_{x_{i}, p_{i}}(\tau)+\log \psi_{x_{i}, p_{i}}(\sigma)^{\chi(\tau)^{-1}} \\
& \quad+\frac{1}{2}\left[\log \psi_{x_{i}, p_{i}}(\tau), \log \psi_{x_{i}, p_{i}}(\sigma)^{\chi(\tau)^{-1}}\right]+\cdots .
\end{aligned}
$$

Now the second part of the proposition follows immediately from the assumptions ii). 
6.1. We shall define filtrations of the Lie algebras $\operatorname{Der}^{*} L(\mathbf{X})$ and $L(\mathbf{X}) \tilde{\times} \operatorname{Der}^{*} L(\mathbf{X})$ associated with the lower central series of $L(\mathbf{X})$.

Let us set

$\operatorname{Der}_{k}^{*} L(\mathbf{X}):=\left\{D \in \operatorname{Der}^{*} L(\mathbf{X}) \mid \forall X_{i} \in \mathbf{X} \exists A_{i} \in \Gamma^{k} L(\mathbf{X}), D\left(X_{i}\right)=\left[X_{i}, A_{i}\right]\right\}$

and

$$
\gamma_{k}\left(L(\mathbf{X}) \tilde{\times} \operatorname{Der}^{*} L(\mathbf{X})\right):=\Gamma^{k} L(\mathbf{X}) \tilde{\times} \operatorname{Der}_{k}^{*} L(\mathbf{X}) .
$$

Lemma 6.1.0. $\quad \operatorname{Der}_{k}^{*} L(\mathbf{X})\left(\right.$ resp. $\left.\gamma_{k}\left(L(\mathbf{X}) \tilde{\times} \operatorname{Der}^{*} L(\mathbf{X})\right)\right)$ is a Lie ideal of $\operatorname{Der}^{*} L(\mathbf{X})$ (resp. $L(\mathbf{X}) \tilde{\times} \operatorname{Der}^{*} L(\mathbf{X})$ ). We have isomorphisms of Lie algebras

$$
\bigoplus_{k=1}^{\infty} \operatorname{Der}_{k}^{*} L(\mathbf{X}) / \operatorname{Der}_{k+1}^{*} L(\mathbf{X})=\operatorname{Der}^{*} \operatorname{Lie}(\mathbf{X})
$$

and

$\bigoplus_{k=1}^{\infty} \gamma_{k}\left(L(\mathbf{X}) \tilde{x} \operatorname{Der}^{*} L(\mathbf{X})\right) / \gamma_{k+1}\left(L(\mathbf{X}) \tilde{\times} \operatorname{Der}^{*} L(\mathbf{X})\right)=\operatorname{Lie}(\mathbf{X}) \tilde{\times} \operatorname{Der}^{*} \operatorname{Lie}(\mathbf{X})$

Proof. The lemma follows from the fact that the graded associated Lie algebra $\bigoplus_{k=1}^{\infty} \Gamma^{k} L(\mathbf{X}) / \Gamma^{k+1} L(\mathbf{X})$ is canonically isomorphic to $\operatorname{Lie}(\mathbf{X})$.

Let $T$ be a finite subset of $\hat{X}(K)^{2}$. We set

$$
\mathfrak{k}^{T}(X):=g r \operatorname{Lie}\left(\mathcal{K}_{1}^{T}(X) / \mathcal{K}_{\infty}^{T}(X)\right):=\bigoplus_{i=1}^{\infty} \operatorname{Lie}\left(\mathcal{K}_{i}^{T}(X) / \mathcal{K}_{i+1}^{T}(X)\right) \otimes \mathbf{Q}
$$

The homomorphism of Lie algebras

$$
\operatorname{Lie}()_{x, p}: \operatorname{Lie}\left(\mathcal{K}_{1}^{T}(X) / \mathcal{K}_{\infty}^{T}(X)\right) \longrightarrow L(\mathbf{X}) \tilde{\times} \operatorname{Der}^{*} L(\mathbf{X})
$$

is compatible with filtrations $\left\{\operatorname{Lie}\left(\mathcal{K}_{i}^{T}(X) / \mathcal{K}_{\infty}^{T}(X)\right)\right\}_{i=1}^{\infty}$ of $\operatorname{Lie}\left(\mathcal{K}_{1}^{T}(X) /\right.$ $\left.\mathcal{K}_{\infty}^{T}(X)\right)$ and $\left\{\gamma_{i}\left(L(\mathbf{X}) \tilde{\times} \operatorname{Der}^{*} L(\mathbf{X})\right)\right\}_{i=1}^{\infty}$ of $L(\mathbf{X}) \tilde{\times} \operatorname{Der}^{*} L(\mathbf{X})$. Therefore it induces a homomorphism of associated graded Lie algebras

$$
\pi_{z, v}^{T}: \mathfrak{k}^{T}(X) \longrightarrow \operatorname{Lie}(\mathbf{X}) \tilde{\times} \operatorname{Der}^{*} \operatorname{Lie}(\mathbf{X})
$$

Let us set

$$
\mathfrak{k}^{T}(X)^{\diamond}:=\bigoplus_{i=1}^{\infty}\left(\operatorname{Lie}\left(\mathcal{K}_{i}^{T}(X) / \mathcal{K}_{i+1}^{T}(X)\right) \otimes \mathbf{Q}\right)^{*}
$$


Then $\mathfrak{k}^{T}(X)^{\diamond}$ is a Lie coalgebra and we have a homomorphism of Lie coalgebras

$$
\left(\pi_{z, v}^{T}\right)^{\diamond}:\left(\operatorname{Lie}(\mathbf{X}) \tilde{\times} \operatorname{Der}^{*} \operatorname{Lie}(\mathbf{X})\right)^{\diamond} \longrightarrow \mathfrak{k}^{T}(X)^{\diamond} .
$$

Moreover an inclusion $S \subset T$ of finite subsets of $\hat{X}(K)^{2}$ induces a morphism of Lie coalgebras

$$
\mathfrak{k}^{S}(X)^{\diamond} \longrightarrow \mathfrak{k}^{T}(X)^{\diamond}
$$

DeFinition 6.1.1. Let $\mathcal{C}$ be the category whose objects are all finite subsets of $\hat{X}(K)^{2}$ and whose morphisms are inclusions. We set

$$
\mathfrak{k}(X)^{\diamond}:=\underset{\mathcal{C}}{\lim } \mathfrak{k}^{T}(X)^{\diamond} .
$$

The $\mathbf{Q}_{l}$-vector space $\mathfrak{k}(X)^{\diamond}$ is a Lie coalgebra and morphisms $\left(\pi_{z, v}^{T}\right)^{\diamond}$ : $\left(\operatorname{Lie}(\mathbf{X}) \tilde{\times} \operatorname{Der}^{*} \operatorname{Lie}(\mathbf{X})\right)^{\diamond} \rightarrow \mathfrak{k}^{T}(X)^{\diamond}$ induce a morphism of Lie coalgebras

$$
\pi_{z, v}^{\diamond}:\left(\operatorname{Lie}(\mathbf{X}) \tilde{\times} \operatorname{Der}^{*} \operatorname{Lie}(\mathbf{X})\right)^{\diamond} \longrightarrow \mathfrak{k}(X)^{\diamond} .
$$

Observe that

$$
\mathcal{L}^{e}(z, v)=e^{*} \circ \pi_{z, v}=\pi_{z, v}^{\diamond}\left(e^{*}\right)
$$

and

$$
\mathcal{L}^{\varepsilon}(v)=\varepsilon \circ \pi_{v, v}=\varepsilon \circ \pi_{z, v}=\pi_{v, v}^{\diamond}(\varepsilon)=\pi_{z, v}^{\diamond}(\varepsilon)
$$

for any $e \in \mathcal{B}$ and for any $\varepsilon \in\left(\operatorname{Der}^{*} \operatorname{Lie}(\mathbf{X})\right)^{\diamond}$ of degree $n$.

Hence we get

$$
d \mathcal{L}^{e}(z, v)=d\left(\pi_{z, v}^{\diamond}\left(e^{*}\right)\right)=\left(\pi_{z, v}^{\diamond} \wedge \pi_{z, v}^{\diamond}\right)\left(d\left(e^{*}\right)\right) .
$$

Warning: $\pi_{z, v}^{\diamond}$ is not injective, hence we can have $d\left(e^{*}\right) \neq 0$ but $d\left(\mathcal{L}^{e}(z, v)\right)=$ 0 .

Proposition 6.1.2. Let $\varphi \in L(\mathbf{X})^{\diamond}$ be a linear form of degree $m$. If

$$
d(\varphi)=\sum_{k+j=m}\left(\sum_{e \in \mathcal{B}_{k}, e^{\prime} \in \mathcal{B}_{j}} c_{e, e^{\prime}} e^{*} \wedge e^{\prime *}+\sum_{e \in \mathcal{B}_{k}, \varepsilon \in\left(\operatorname{Der}^{*} L(\mathbf{X})\right)^{j}} b_{e, \varepsilon} e^{*} \wedge \varepsilon\right)
$$

in $\bigwedge^{2}\left(L(\mathbf{X}) \tilde{\times} \operatorname{Der}^{*} L(\mathbf{X})\right)^{\diamond}$ then

$$
\begin{aligned}
d\left(\mathcal{L}^{\varphi}(z, v)\right)=\sum_{k+j=m}\left(\sum_{e \in \mathcal{B}_{k}, e^{\prime} \in \mathcal{B}_{j}} c_{e, e^{\prime}} \mathcal{L}^{e}(z, v) \wedge \mathcal{L}^{e^{\prime}}(z, v)\right. & \\
+ & \left.\sum_{e \in \mathcal{B}_{k}, \varepsilon \in\left(\operatorname{Der}^{*} L(\mathbf{X})\right)^{j}} b_{e, \varepsilon} \mathcal{L}^{e}(z, v) \wedge \mathcal{L}^{\varepsilon}(v)\right)
\end{aligned}
$$

in $\mathfrak{k}(X)^{\diamond}$. 


\section{$\S 7$. Analog of Zagier conjecture}

7.0. We shall present here a conjecture which is an $l$-adic analog of conjectures concerning iterated integrals from [W3]. These conjectures are generalizations of the Zagier conjecture for classical complex polylogarithms. The main ideas come from the Deligne-Beilinson paper.

We assume that there exists a category of mixed Tate motives over Spec $K$ such as in [BD]. (We do not know if recent constructions of Voevodsky and others are sufficient for our purpose.) We shall denote this category by $\mathcal{M M}_{K}$. The category $\mathcal{M M}_{K}$ is a tannakian category and it is equivalent to a category of representations of a pro-algebraic group $\Pi_{K}$ defined over $\mathbf{Q}$. Let $U_{K}:=\operatorname{ker}\left(\Pi_{K} \rightarrow \mathbf{G}_{\mathbf{m}}\right)$. The group $U_{K}$ is a pro-algebraic pro-unipotent group defined over $\mathbf{Q}$. We denote by Lie $U_{K}$ its Lie algebra. This Lie algebra is equipped with the weight filtration. Let

$$
\text { Lie } U_{K}=\bigoplus_{n=1}^{\infty}\left(\mathcal{L} \text { ie } U_{K}\right)_{n}
$$

be the associated graded Lie algebra. We set

$$
\left(\mathcal{L} \text { ie } U_{K}\right)^{\diamond}:=\bigoplus_{n=1}^{\infty}\left(\mathcal{L} i e U_{K}\right)_{n}^{\diamond}
$$

$\left(\mathcal{L} \text { ie } U_{K}\right)^{\diamond}$ equipped with $d$ - the dual of the Lie bracket - is a Lie coalgebra.

Let $X$ be a projective line over $K$ minus a finite number of $K$-points. We shall construct a Lie subcoalgebra of the Lie coalgebra $\left(\mathcal{L} i e U_{K}\right)^{\diamond}$ corresponding to the pro-unipotent part of the fundamental group of the tannakian category generated by mixed motives of torsors of paths from $v$ to $z$ on $X$ for all pairs $(z, v) \in \hat{X}(K)^{2}$.

We shall construct this Lie subcoalgebra of $\left(\mathcal{L} i e U_{K}\right)^{\diamond}$ in the inductive way. This Lie subcoalgebra will be a graded Lie coalgebra. The construction in degree 1 will be clear. We shall assume that we have constructed our Lie coalgebra up to degree $N$, i.e., we have $\bigoplus_{i=1}^{N-1} \mathcal{L}_{i}$ and $d: \bigoplus_{i=1}^{N-1} \mathcal{L}_{i} \rightarrow$ $\bigwedge^{2}\left(\bigoplus_{i=1}^{N-2} \mathcal{L}_{i}\right)$.

We construct a candidate $\mathcal{L}_{N}^{\prime}$ in degree $N$ and $d_{N}^{\prime}: \mathcal{L}_{N}^{\prime} \rightarrow \bigwedge^{2}\left(\bigoplus_{i=1}^{N-1} \mathcal{L}_{i}\right)$. Our construction should be motivic hence we should have the following com- 
mutative diagram

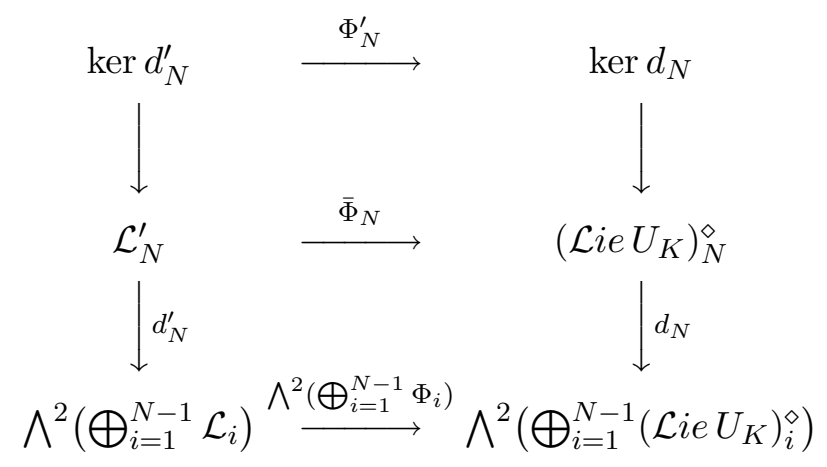

Then it is clear that $\mathcal{L}_{N}=\mathcal{L}_{N}^{\prime} / \operatorname{ker} \bar{\Phi}_{N}$. Observe that $\mathcal{L}_{N}^{\prime} / \operatorname{ker} \bar{\Phi}_{N}=$ $\mathcal{L}_{N}^{\prime} / \operatorname{ker} \Phi_{N}^{\prime}$. In fact we shall conjecture that we have a map

$$
\Phi_{N}^{\prime}: \operatorname{ker} d_{N}^{\prime} \longrightarrow \operatorname{ker} d_{N}=\operatorname{Ext}_{\mathcal{M} \mathcal{M}_{K}}^{1}(\mathbf{Q}(0), \mathbf{Q}(N)) \otimes \mathbf{Q}
$$

and we shall set $\mathcal{L}_{N}=\mathcal{L}_{N}^{\prime} / \operatorname{ker} \Phi_{N}^{\prime}$. This is a short motivic justification of our next steps.

We recall that in the category $\mathcal{M M}_{K}$

$$
\operatorname{Ext}_{\mathcal{M} \mathcal{M}_{K}}^{1}(\mathbf{Q}(0), \mathbf{Q}(1)) \otimes \mathbf{Q}=K^{*} \otimes \mathbf{Q} .
$$

7.1. Let $X=\mathbf{P}_{K}^{1} \backslash\left\{a_{1}, \ldots, a_{n+1}\right\}$. We assume for simplicity that $a_{n+1}=\infty$. Let us choose a tangential base point $v_{i}$ (a tangent vector) at $a_{i}$ for $i=1,2, \ldots, n+1$. Let $\mathcal{B}$ be a Hall base of $\operatorname{Lie}(\mathbf{X})$ and let $\mathcal{B}_{m}$ be elements of degree $m$ in $\mathcal{B}$.

For $k=1$ we set $\mathcal{L}_{1}:=K^{*} \otimes \mathbf{Q}, d_{1}=0: \mathcal{L}_{1} \rightarrow 0$. We define symbols $\{z, v\}_{X_{i}} \in \mathcal{L}_{1}$ in the following way. If $(z, v) \in X(K)^{2}$ then $\{z, v\}_{X_{i}}:=$ $\frac{z-a_{i}}{v-a_{i}} \otimes 1 \in \mathcal{L}_{1}$, if $z \in X(K)$ and $v=\overrightarrow{a_{i} x}$ then $\{z, v\}_{X_{i}}:=\frac{z-a_{i}}{x-a_{i}} \otimes 1 \in \mathcal{L}_{1}$ and $\{z, v\}_{X_{j}}:=\frac{z-a_{j}}{a_{i}-a_{j}} \otimes 1 \in \mathcal{L}_{1}$, if $z=\overrightarrow{a_{k} x^{\prime}}$ and $v=\overrightarrow{a_{l} x}$ then $\{z, v\}_{X_{i}}:=\frac{a_{k}-a_{i}}{a_{l}-a_{i}} \otimes$ $1 \in \mathcal{L}_{1}$ for $i \neq k, l,\{z, v\}_{X_{k}}:=\frac{x^{\prime}-a_{k}}{a_{l}-a_{k}} \otimes 1 \in \mathcal{L}_{1}$ and $\{z, v\}_{X_{l}}:=\frac{a_{k}-a_{l}}{x-a_{l}} \otimes 1 \in \mathcal{L}_{1}$.

We define a map

$$
\varphi_{1}: \mathcal{L}_{1} \longrightarrow \operatorname{Ext}_{\mathcal{M} \mathcal{M}_{K}}^{1}(\mathbf{Q}(0), \mathbf{Q}(1)) \otimes \mathbf{Q}=K^{*} \otimes \mathbf{Q}
$$

by $\varphi_{1}(z \otimes 1):=z \otimes 1$. We define

$$
\psi_{1}: \mathcal{L}_{1} \longrightarrow H^{1}\left(\mathcal{K}_{1}(X), \mathbf{Q}_{l}(1)\right)
$$

by $\psi_{1}\left(\{z, v\}_{X_{i}}\right):=\mathcal{L}^{X_{i}}(z, v)$. (We recall that $\mathcal{K}_{1}(X)=\operatorname{Gal}\left(\bar{K} / K\left(\mu_{l^{\infty}}\right)\right)$.) 
Proposition 7.1.0. The diagram

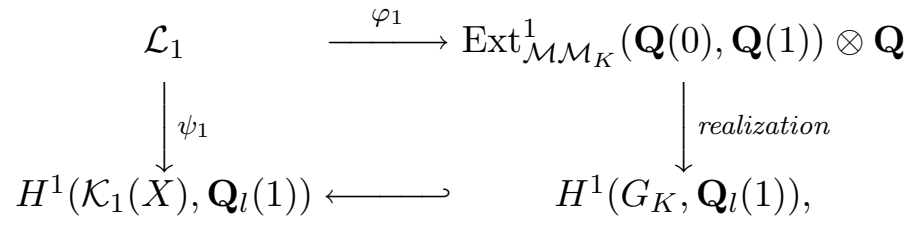

commutes, where realization associates to $z \otimes 1 \in K^{*} \otimes \mathbf{Q}=\operatorname{Ext}_{\mathcal{M} \mathcal{M}_{K}}^{1}(\mathbf{Q}(0)$, $\mathbf{Q}(1)) \otimes \mathbf{Q}$ the Kummer character corresponding to $z$.

Proof. Let $(z, v) \in \hat{X}(K)^{2}$ and let $p$ be a path from $v$ to $z$. First we consider the case when $z$ and $v$ are $K$-points of $X$. Let us take $\sigma \in G_{K\left(\mu_{l} \infty\right)}$. We shall calculate the coefficient of $\left(\log \sigma_{x, p}\right)(1)$ at $X_{i}$. This coefficient is equal to the exponent of $\mathfrak{f}_{p}(\sigma)$ at $x_{i}$. Let $\zeta$ be a coordinate on $\mathbf{P}_{K}^{1}$. The loop $\mathfrak{f}_{p}(\sigma)=p^{-1} \cdot \sigma \cdot p \cdot \sigma^{-1}$ transforms $\left(\zeta-a_{i}\right)^{1 / l^{n}}$ into

$$
\frac{\sigma^{-1}\left(\left(v-a_{i}\right)^{1 / l^{n}}\right)}{\left(v-a_{i}\right)^{1 / l^{n}}} \cdot \frac{\sigma\left(\left(z-a_{i}\right)^{1 / l^{n}}\right)}{\left(z-a_{i}\right)^{1 / l^{n}}} \cdot\left(\zeta-a_{i}\right)^{1 / l^{n}} .
$$

This finishes the proof of the proposition when $(z, v) \in X(K)^{2}$. Now we assume that $v=\overrightarrow{a_{i} x}$ is a tangential base point and $z$ is a $K$-point. The isomorphism of $\mathbf{P}_{K}^{1}$ given by $y \rightarrow \frac{y-a_{i}}{x-a_{i}}$ is defined over $K$. Hence we can assume that $a_{i}=0, v=\overrightarrow{01}$ and $p$ is a path from $\overrightarrow{01}$ to $z_{1}=\frac{z-a_{i}}{x-a_{i}}$. Let $\zeta$ be a local parameter corresponding to the tangential base point $\overrightarrow{01}$. The loop $\mathfrak{f}_{p}(\sigma)$ transforms $\zeta^{1 / l^{n}}$ into $\sigma\left(\left(\frac{z-a_{i}}{x-a_{i}}\right)^{1 / l^{n}}\right) \cdot\left(\left(\frac{z-a_{i}}{x-a_{i}}\right)^{1 / l^{n}}\right)^{-1} \cdot \zeta^{1 / l^{n}}$. Hence the exponent of $\mathfrak{f}_{p}(\sigma)$ at $x_{i}$ is equal to the Kummer character of $\frac{z-a_{i}}{x-a_{i}}$ evaluated at $\sigma$. The other cases we left to the readers.

Let $N>1$. We assume that the groups $\mathcal{L}_{k}$, the symbols $\{z, v\}_{e} \in \mathcal{L}_{k}$ for $e \in \mathcal{B}_{k}$, the homomorphisms $d_{k}: \mathcal{L}_{k} \rightarrow \bigoplus_{i+j=k} \mathcal{L}_{i} \wedge \mathcal{L}_{j}, \varphi_{k}: \operatorname{ker} d_{k} \rightarrow$ $\operatorname{Ext}_{\mathcal{M} \mathcal{M}_{K}}^{1}(\mathbf{Q}(0), \mathbf{Q}(k)) \otimes \mathbf{Q}$ and $\psi_{k}: \mathcal{L}_{k} \rightarrow H_{\mathcal{C}}^{1}\left(\mathcal{K}_{k}(X), \mathbf{Q}_{l}(k)\right)$ are defined for $k<N$. We assume that for $k<N$ the diagram

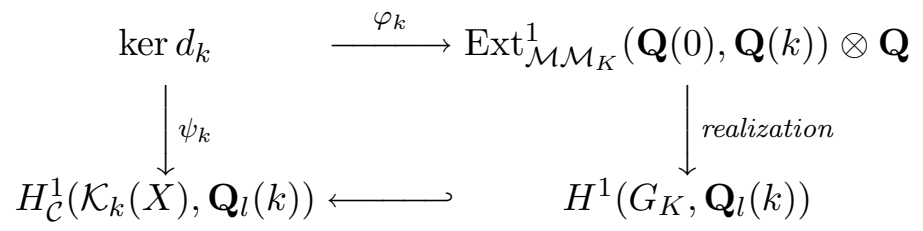


commutes. We recall that the lower horizontal morphism is injective by Lemma 3.0.10.

Let $\varphi \in \operatorname{Lie}(\mathbf{X})^{\diamond}$ be a linear form of degree $k$ defined over $\mathbf{Q}$. If $\varphi=$ $\sum_{i} a_{i}\left(e_{i}^{k}\right)^{*}$ then we set $\{z, v\}_{\varphi}:=\sum_{i} a_{i}\{z, v\}_{e_{i}^{k}}$.

Let $\varepsilon \in\left(\operatorname{Der}^{*} \operatorname{Lie}(\mathbf{X})\right)^{\diamond}=\bigoplus_{i=1}^{n}\left(\operatorname{Lie}(\mathbf{X}) /\left\langle X_{i}\right\rangle\right)^{\diamond}$ be a linear form of degree $k$ defined over $\mathbf{Q}$. Assume that $\varepsilon=\left(\varphi_{1}, \ldots, \varphi_{n}\right)$, where $\varphi_{i} \in$ $\left(L(\mathbf{X}) /\left\langle X_{i}\right\rangle\right)^{\diamond}$. Then we set

$$
\{v\}_{\varepsilon}:=\sum_{i=1}^{n}\left\{v_{i}, v\right\}_{\varphi_{i}}
$$

Observe that $\mathcal{L}^{\varepsilon}(v)=\sum_{i=1}^{n} \mathcal{L}^{\varphi_{i}}\left(v_{i}, v\right)$.

Let $\mathcal{B}_{N}=\left\{e_{i}^{N}\right\}_{i \in I}$. For each $e_{i}^{N}$ we set

$\mathcal{L}^{e_{i}^{N}}:=\bigoplus_{\{z, v\} \in \hat{X}(K)^{2}} \mathbf{Q}\{z, v\}_{e_{i}^{N}}^{\prime}-$ a vector space over $\mathbf{Q}$ on symbols $\{z, v\}_{e_{i}^{N}}^{\prime}$

and

$$
\mathcal{L}_{N}^{\prime}:=\bigoplus_{i \in I} \mathcal{L}^{e_{i}^{N}}
$$

Let $e \in \mathcal{B}_{N}$. We define

$$
d_{N}^{\prime}: \mathcal{L}_{N}^{\prime} \longrightarrow \bigoplus_{i+j=N} \mathcal{L}_{i} \wedge \mathcal{L}_{j}
$$

setting

$$
\begin{aligned}
d_{N}^{\prime}\left(\{z, v\}_{e}^{\prime}\right)= & \sum_{k+j=N}\left(\sum_{e_{1} \in \mathcal{B}_{k}, e_{2} \in \mathcal{B}_{j}} c_{e_{1}, e_{2}}\{z, v\}_{e_{1}} \wedge\{z, v\}_{e_{2}}\right. \\
& \left.+\sum_{e^{\prime} \in \mathcal{B}_{k}, \varepsilon \in\left(\operatorname{Der}^{*} L(\mathbf{X})\right)^{j}} b_{e^{\prime}, \varepsilon}\{z, v\}_{e^{\prime}} \wedge\{v\}_{\varepsilon}\right)
\end{aligned}
$$

if

$$
d\left(e^{*}\right)=\sum_{k+j=N}\left(\sum_{e_{1} \in \mathcal{B}_{k}, e_{2} \in \mathcal{B}_{j}} c_{e_{1}, e_{2}} e_{1}^{*} \wedge e_{2}^{*}+\sum_{e^{\prime} \in \mathcal{B}_{k}, \varepsilon \in\left(\operatorname{Der}^{*} L(\mathbf{X})\right)^{j}} b_{e^{\prime}, \varepsilon} e^{*} \wedge \varepsilon\right)
$$

in $\bigwedge^{2}\left(\operatorname{Lie}(\mathbf{X}) \tilde{\times} \operatorname{Der}{ }^{*} \operatorname{Lie}(\mathbf{X})\right)^{\diamond}$ 
Conjecture $D_{N}$. There is a homomorphism

$$
\varphi_{N}^{\prime}: \operatorname{ker} d_{N}^{\prime} \longrightarrow \operatorname{Ext}_{\mathcal{M} \mathcal{M}_{K}}^{1}(\mathbf{Q}(0), \mathbf{Q}(N)) \otimes \mathbf{Q}
$$

such that the diagram

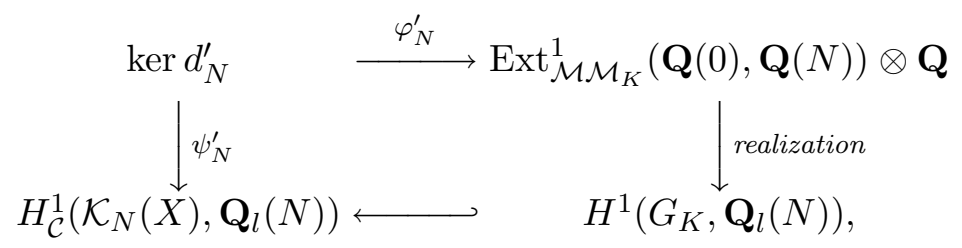

commutes, where the map $\psi_{N}^{\prime}$ is given by $\psi_{N}^{\prime}\left(\{z, v\}_{e_{i}^{N}}^{\prime}\right):=\mathcal{L}^{e_{i}^{N}}(z, v)$.

If the conjecture is true then we set $\mathcal{L}_{N}:=\mathcal{L}_{N}^{\prime} / \operatorname{ker} \varphi_{N}^{\prime}$. The maps $d_{N}$, $\psi_{N}$ and $\varphi_{N}$ are defined by passing to quotient. The symbol $\{z, v\}_{e_{i}^{N}}$ is the image of $\{z, v\}_{e_{i}^{N}}^{\prime}$ in $\mathcal{L}_{N}$.

Definition 7.1.1. We set

$$
\mathcal{L}^{K}(X):=\bigoplus_{N=1}^{\infty} \mathcal{L}_{N} .
$$

We define $d: \mathcal{L}^{K}(X) \rightarrow \mathcal{L}^{K}(X) \wedge \mathcal{L}^{K}(X)$ by setting $\left.d\right|_{\mathcal{L}_{N}}:=d_{N}$.

Lemma 7.1.2. Let $\varepsilon \in\left(\operatorname{Der}^{*} \operatorname{Lie}(\mathbf{X})\right)^{\diamond}$ be a linear form of degree $N$ defined over $\mathbf{Q}$. Assume that

$$
d \varepsilon=\sum_{p+q=N} \sum_{\varepsilon_{1} \in\left(\operatorname{Der}{ }^{*} \operatorname{Lie}(\mathbf{X})\right)^{p}, \varepsilon_{2} \in(\operatorname{Der} * \operatorname{Lie}(\mathbf{X}))^{q}} a_{\varepsilon_{1}, \varepsilon_{2}} \varepsilon_{1} \wedge \varepsilon_{2}
$$

in $\bigwedge^{2}\left(\operatorname{Der}^{*} \operatorname{Lie}(\mathbf{X})\right)^{\diamond}$. Then

$$
d\left(\{v\}_{\varepsilon}\right)=\sum_{p+q=N} \sum_{\varepsilon_{1} \in\left(\operatorname{Der}^{*} \operatorname{Lie}(\mathbf{X})\right)^{p}, \varepsilon_{2} \in\left(\operatorname{Der}^{*} \operatorname{Lie}(\mathbf{X})\right)^{q}} a_{\varepsilon_{1}, \varepsilon_{2}}\{v\}_{\varepsilon_{1}} \wedge\{v\}_{\varepsilon_{2}} .
$$

Proof. We recall that

$\operatorname{Der}^{*} \operatorname{Lie}(\mathbf{X})=\{D \in \operatorname{Der} \operatorname{Lie}(\mathbf{X}) \mid$

$$
\left.\forall X_{i} \in \mathbf{X} \exists A_{i} \in \operatorname{Lie}(\mathbf{X}), D\left(X_{i}\right)=\left[X_{i}, A_{i}\right]\right\} .
$$


The derivation $D \in \operatorname{Der}^{*}(\operatorname{Lie}(\mathbf{X}))$ such that $D\left(X_{i}\right)=\left[X_{i}, A_{i}\right]$ we shall denote by $D_{\left(A_{1}, \ldots, A_{n}\right)}=D_{\left(A_{i}\right)_{i=1, \ldots, n}}$. We have an identification

$$
\operatorname{Der}^{*} \operatorname{Lie}(\mathbf{X})=\bigoplus_{i=1}^{n} \operatorname{Lie}(\mathbf{X}) /\left\langle X_{i}\right\rangle
$$

sending $D_{\left(A_{1}, \ldots, A_{n}\right)}$ to a sequence $\left(A_{1}, \ldots, A_{n}\right)$. One easily checks that (7.1.3)

$$
\left[D_{\left(V_{k}\right)_{k=1, \ldots, n}}, D_{\left(W_{k}\right)_{k=1, \ldots, n}}\right]=D_{\left(\left[V_{k}, W_{k}\right]+D_{\left(V_{j}\right)_{j}}\left(W_{k}\right)-D_{\left(W_{j}\right)_{j}}\left(V_{k}\right)\right)_{k=1, \ldots, n}} .
$$

If $e \in \mathcal{B}$ then we set $(e)^{i}=\left(a_{1}, \ldots, a_{n}\right) \in \bigoplus_{k=1}^{n} \operatorname{Lie}(\mathbf{X}) /\left\langle X_{k}\right\rangle$, where $a_{i}=e$ and $a_{j}=0$ for $j \neq i$.

Let $\varepsilon \in\left(\operatorname{Der}^{*} \operatorname{Lie}(\mathbf{X})\right)^{\diamond}$. Then $\varepsilon=\sum_{i=1}^{n}\left(\sum_{e \in \mathcal{B}} n_{e, i}(e)^{i *}\right)$, where $(e)^{i *}$ is a composition of $e^{*}$ with the projection $\bigoplus_{k=1}^{n} \operatorname{Lie}(\mathbf{X}) /\left\langle X_{k}\right\rangle \rightarrow \operatorname{Lie}(\mathbf{X}) /\left\langle X_{i}\right\rangle$. We shall compare $d\left(e^{*}\right)$ with $d\left((e)^{i *}\right)$ in $\left(\operatorname{Lie}(\mathbf{X}) \tilde{\times} \operatorname{Der}^{*} \operatorname{Lie}(\mathbf{X})\right)^{\diamond}$. Observe that $e^{*} \in \operatorname{Lie}(\mathbf{X})^{\diamond}$ and $(e)^{i *} \in\left(\operatorname{Der}{ }^{*} \operatorname{Lie}(\mathbf{X})\right)^{\diamond}$. It follows from the definition of the Lie bracket in the semi-direct product $\operatorname{Lie}(\mathbf{X}) \tilde{\times} \operatorname{Der}^{*}(\operatorname{Lie}(\mathbf{X}))$ that

$$
d\left(e^{*}\right)=\sum_{e_{1}, e_{2} \in \mathcal{B}} e^{*}\left(\left[e_{1}, e_{2}\right]\right) e_{1}^{*} \wedge e_{2}^{*}+\sum_{k=1}^{n} \sum_{e_{3}, e_{4} \in \mathcal{B}} e^{*}\left(D_{\left(e_{4}\right)^{k}}\left(e_{3}\right)\right) e_{3}^{*} \wedge\left(e_{4}\right)^{k *} .
$$

Hence we get

$$
\begin{aligned}
d\left(\left\{v_{i}, v\right\}_{e}\right)= & \sum_{e_{1}, e_{2} \in \mathcal{B}} e^{*}\left(\left[e_{1}, e_{2}\right]\right)\left\{v_{i}, v\right\}_{e_{1}} \wedge\left\{v_{i}, v\right\}_{e_{2}} \\
& \quad+\sum_{k=1}^{n} \sum_{e_{3}, e_{4} \in \mathcal{B}} e^{*}\left(D_{\left(e_{4}\right)^{k}}\left(e_{3}\right)\right)\left\{v_{i}, v\right\}_{e_{3}} \wedge\{v\}_{\left(e_{4}\right)^{k *}}
\end{aligned}
$$

On the other side it follows from (7.1.3) that

$$
\begin{aligned}
d\left((e)^{i *}\right)=\sum_{e_{1}, e_{2} \in \mathcal{B}} e^{*}\left(\left[e_{1}, e_{2}\right]\right)\left(e_{1}\right)^{i *} \wedge\left(e_{2}\right)^{i *} & \\
& +\sum_{k=1}^{n} \sum_{e_{3}, e_{4} \in \mathcal{B}} e^{*}\left(D_{\left(e_{4}\right)^{k}}\left(e_{3}\right)\right)\left(e_{3}\right)^{i *} \wedge\left(e_{4}\right)^{k *} .
\end{aligned}
$$

We recall that we have defined $\{v\}_{(e)^{i *}}:=\left\{v_{i}, v\right\}_{e}$. Hence if in the right hand side of the equality (7.1.6) we replace $\left(e_{\alpha}\right)^{j *}$ by $\{v\}_{\left(e_{\alpha}\right)^{j *}}$ then we get 
the right hand side of the equality (7.1.5). Therefore the lemma is proved for $\varepsilon=(e)^{i *}$. Any $\varepsilon \in\left(\operatorname{Der}^{*} \operatorname{Lie}(\mathbf{X})\right)^{\diamond}$ is a linear combination of $(e)^{i *}$, hence the lemma is proved for any $\varepsilon \in\left(\operatorname{Der}^{*} \operatorname{Lie}(\mathbf{X})\right)^{\diamond}$.

Proposition 7.1.7. The $\mathbf{Q}$-vector space $\mathcal{L}^{K}(X)$ equipped with the homomorphism $d: \mathcal{L}^{K}(X) \rightarrow \mathcal{L}^{K}(X) \wedge \mathcal{L}^{K}(X)$ is a Lie coalgebra.

Proof. It is enough to show that

$$
\sum_{i=0}^{2} \sigma^{i} \circ\left(d \otimes i d_{\mathcal{L}^{K}(X)}\right) \circ d=0,
$$

where $\sigma(a \otimes b \otimes c)=b \otimes c \otimes a$. In the Lie coalgebra $(\operatorname{Lie}(\mathbf{X}) \tilde{\times} \operatorname{Der} * \operatorname{Lie}(\mathbf{X}))^{\diamond}$ we obviously have

$$
\sum_{i=0}^{2} \sigma^{i} \circ\left(d \otimes i d_{\left(\operatorname{Lie}(\mathbf{X}) \tilde{\times} \operatorname{Der}^{*} \operatorname{Lie}(\mathbf{X})\right)^{\diamond}}\right) \circ d=0 .
$$

The calculation of $d\left(\{z, v\}_{e}\right)$ (corresponding to $d\left(e^{*}\right)$ in $\left(\operatorname{Lie}(\mathbf{X}) \tilde{\times} \operatorname{Der}^{*}\right.$ $\left.\operatorname{Lie}(\mathbf{X}))^{\diamond}\right)$ involves only symbols $\{z, v\}_{e_{1}}$ (corresponding to $e_{1}^{*}$ in $(\operatorname{Lie}(\mathbf{X}) \tilde{x}$ $\left.\left.\operatorname{Der}{ }^{*} \operatorname{Lie}(\mathbf{X})\right)^{\diamond}\right)$ and $\left\{v_{i}, v\right\}_{e_{2}}=\{v\}_{\left(e_{2}\right)^{i *}}\left(\right.$ corresponding to $\left(e_{2}\right)^{i *}$ in $(\operatorname{Lie}(\mathbf{X}) \tilde{\times}$ $\left.\left.\operatorname{Der}^{*} \operatorname{Lie}(\mathbf{X})\right)^{\diamond}\right)$. Hence the proposition follows from Lemma 7.1.2.

Proposition 7.1.10. Assume that Conjectures $D_{N}$ are true for all $N$ and that for all $N$ the maps realization : $\operatorname{Ext}_{\mathcal{M} \mathcal{M}_{K}}^{1}(\mathbf{Q}(0), \mathbf{Q}(N)) \otimes \mathbf{Q} \rightarrow$ $H^{1}\left(G_{K}, \mathbf{Q}_{l}(N)\right)$ are injective. Let $\left(z_{i}, v_{i}\right) \in \hat{X}(K)^{2}$ and let $e_{i}^{N} \in \mathcal{B}_{N}$ for $i=1, \ldots, m$. Let $n_{i} \in \mathbf{Q}_{l}$ for $i=1, \ldots, m$. Then $\sum_{i=1}^{m} n_{i} \mathcal{L}^{e_{i}^{N}}\left(z_{i}, v_{i}\right)=0$ if and only if $\sum_{i=1}^{m} n_{i}\left\{z_{i}, v_{i}\right\}_{e_{i}^{N}}=0$ in $\mathcal{L}_{N} \otimes \mathbf{Q}_{l}$.

Proof. It is well known that the restriction map $H^{1}\left(G_{K}, \mathbf{Q}_{l}(1)\right) \rightarrow$ $H^{1}\left(G_{K\left(\mu_{l} \infty\right)}, \mathbf{Q}_{l}(1)\right)$ is injective. Hence it follows from Proposition 7.1.0 that the proposition is true for $k=1$. Let us assume that it is true for $k<N$. Let $\sum_{i=1}^{m} n_{i} \mathcal{L}^{e_{i}^{N}}\left(z_{i}, v_{i}\right)=0$. This implies that $d\left(\sum_{i=1}^{m} n_{i} \mathcal{L}^{e_{i}^{N}}\left(z_{i}, v_{i}\right)\right)=$ $\sum_{k+l=N} \sum_{\alpha, \beta} c_{\alpha, \beta}^{k, l} \mathcal{L}^{e_{\alpha}^{k}}\left(z_{\alpha}, v_{\alpha}\right) \cdot \mathcal{L}^{e_{\beta}^{l}}\left(z_{\beta}, v_{\beta}\right)=0$ in $\mathfrak{t}(X)^{\diamond} \wedge \mathfrak{t}(X)^{\diamond}$. Hence for any $\sigma \in \mathcal{K}_{k}^{T}(X) / \mathcal{K}_{k+1}^{T}(X)$ we have $\sum_{\alpha, \beta} c_{\alpha, \beta}^{k, l} \mathcal{L}^{e_{\alpha}^{k}}\left(z_{\alpha}, v_{\alpha}\right)(\sigma) \cdot \mathcal{L}^{e_{\beta}^{l}}\left(z_{\beta}, v_{\beta}\right)=0$ for $T$ sufficiently big. Hence by the induction hypothesis we have $\sum_{\alpha, \beta} c_{\alpha, \beta}^{k, l}$ $\mathcal{L}^{e_{\alpha}^{k}}\left(z_{\alpha}, v_{\alpha}\right)(\sigma) \cdot\left\{z_{\beta}, v_{\beta}\right\}_{e_{\beta}^{l}}=0$. Let $f: \mathcal{L} \rightarrow \mathbf{Q}_{l}$ be a homomorphism. We get that for all $\sigma \in \mathcal{K}_{k}^{T}(X) / \mathcal{K}_{k+1}^{T}(X), \sum_{\alpha, \beta} c_{\alpha, \beta}^{k, l} \mathcal{L}^{e_{\alpha}^{k}}\left(z_{\alpha}, v_{\alpha}\right)(\sigma) \cdot f\left(\left\{z_{\beta}, v_{\beta}\right\}_{e_{\beta}^{l}}\right)=$ 
0 . The induction hypothesis implies that for any homomorphism $f: \mathcal{L} \rightarrow$ $\mathbf{Q}_{l}$ we have $\sum_{\alpha, \beta} c_{\alpha, \beta}^{k, l}\left\{z_{\alpha}, v_{\alpha}\right\}_{e_{\alpha}^{k}} \cdot f\left(\left\{z_{\beta}, v_{\beta}\right\}_{e_{\beta}^{l}}\right)=0$. This implies that $d\left(\sum_{i=1}^{m} n_{i}\left\{z_{i}, v_{i}\right\}_{e_{i}^{N}}\right)=0$. The assumption that the realization and the restriction are injective implies that $\sum_{i=1}^{m} n_{i}\left\{z_{i}, v_{i}\right\}_{e_{i}^{N}}=0$ in $\mathcal{L}^{K}(X) \otimes \mathbf{Q}_{l}$.

Corollary 7.1.11. Assume that Conjectures $D_{N}$ are true for all $N$. Assume that for all $N$ the maps realization: $\operatorname{Ext}_{\mathcal{M} \mathcal{M}_{K}}^{1}(\mathbf{Q}(0), \mathbf{Q}(N)) \otimes \mathbf{Q} \rightarrow$ $H^{1}\left(G_{K}, \mathbf{Q}_{l}(N)\right)$ are injective. Let $q_{i} \in \mathbf{Q}$ for $i=1, \ldots, m$.

i) We have a relation $\sum_{i=1}^{m} q_{i} \mathcal{L}^{e_{i}}\left(z_{i}, v_{i}\right)=0$ if and only if $\sum_{i=1}^{m} q_{i}\left\{z_{i}, v_{i}\right\}_{e_{i}}$ $=0$ in $\mathcal{L}^{K}(X)$.

ii) The vector space of linear relations between functions $\mathcal{L}^{e}(z, v)$ is defined over $\mathbf{Q}$.

Proof. The first part follows immediately from Proposition 7.1.10. Observe that a vector space of linear relations between elements $\{z, v\}_{e}$ is generated by relations with $\mathbf{Q}$-coefficients. This implies the second part of the corollary.

Proposition 7.1.12. Assume that Conjectures $D_{N}$ are true for all $N$. Assume that for all $N$ the maps realization: $\operatorname{Ext}_{\mathcal{M} \mathcal{M}_{K}}^{1}(\mathbf{Q}(0), \mathbf{Q}(N)) \otimes \mathbf{Q} \rightarrow$ $H^{1}\left(G_{K}, \mathbf{Q}_{l}(N)\right)$ are injective. Then the Lie coalgebras $\left(\mathcal{L}^{K}(X) \otimes \mathbf{Q}_{l}, d\right)$ and $\left(\mathfrak{k}(X)^{\diamond}, d\right)$ are isomorphic.

Proof. Let us define a map

$$
r_{l}: \mathcal{L}^{K}(X) \otimes \mathbf{Q}_{l} \longrightarrow \mathfrak{k}(X)^{\diamond}
$$

by $r_{l}\left(\{z, v\}_{e} \otimes 1\right):=\mathcal{L}^{e}(z, v)$. The vector space $\mathfrak{k}(X)^{\diamond}$ is generated over $\mathbf{Q}_{l}$ by linear forms $\mathcal{L}^{e}(z, v)((z, v) \in \hat{X}(K) \times \hat{X}(K), e \in \mathcal{B})$. Corollary 7.1.11 implies that the map $r_{l}$ is an isomorphism of vector spaces over $\mathbf{Q}_{l}$. It follows from the definition of $d$ in $\mathcal{L}^{K}(X)$ that $r_{l}$ is an isomorphism of Lie coalgebras over $\mathbf{Q}_{l}$.

\section{§8. Primitive example in the case $\mathbf{P}^{1} \backslash\{0,1, \infty\}$}

8.0. We shall show here that the functions $a_{x, p}^{\varphi}$ are generalizations of characters considered by Soulé, Deligne, Ihara (see [S1], [S2], [D] and [I1]).

Let $V=P_{\mathbf{Q}}^{1} \backslash\{0,1, \infty\}$. Let us fix a path $p$ from $\overrightarrow{01}$ to $\overrightarrow{10}$. We recall that $\pi_{1}\left(V_{\overline{\mathbf{Q}}}, \overrightarrow{01}\right)$ is a free group on $x$ - a small loop around 0 , and $y$ - a loop 


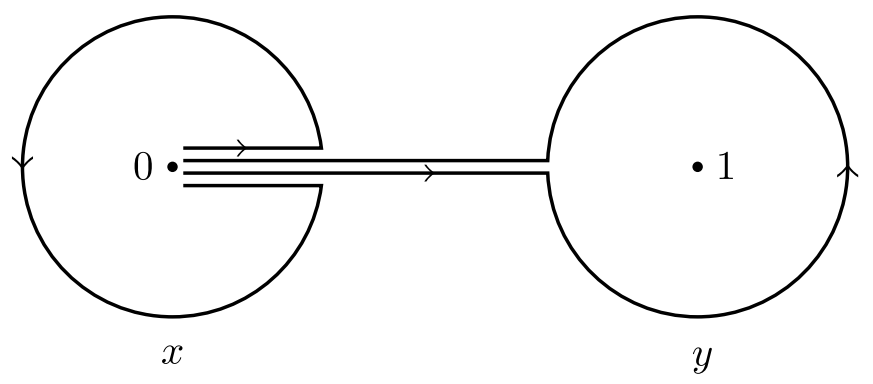

Picture 4

around 1. (One goes from $\overrightarrow{01}$ to $\overrightarrow{10}$ along $p$, makes a small loop around 1 and returns to $\overrightarrow{01}$ along $p$ (see Picture 4).)

The action of $\sigma \in G_{Q\left(\mu_{l} \infty\right)}$ is given by

$$
\sigma(x)=x \quad \text { and } \quad \sigma(y)=\mathfrak{f}_{p}(\sigma)^{-1} \cdot y \cdot \mathfrak{f}_{p}(\sigma) .
$$

Let us set $\pi_{1}^{\prime}:=\left[\pi_{1}\left(V_{\overline{\mathbf{Q}}}, \overrightarrow{01}\right), \pi_{1}\left(V_{\overline{\mathbf{Q}}}, \overrightarrow{01}\right)\right]$ and $\pi_{1}^{\prime \prime}:=\left[\pi_{1}^{\prime}, \pi_{1}^{\prime}\right]$. The element $\mathfrak{f}_{p}(\sigma)$ belongs to $\pi_{1}^{\prime}$. Assume that

$$
\mathfrak{f}_{p}(\sigma)=\prod_{i, j \geq 1}\left(y^{j-1}\left(x^{i-1}(x, y) \cdots\right) \cdots\right)^{\alpha_{i, j}(\sigma)} \bmod \pi_{1}^{\prime \prime} .
$$

It implies that

$$
\sigma((x, y))=(x, y) \prod_{i, j \geq 1}\left(y^{j}\left(x^{i}(x, y) \cdots\right) \cdots\right)^{\alpha_{i, j}(\sigma)} \bmod \pi_{1}^{\prime \prime} .
$$

Ihara shows that $\pi_{1}^{\prime} / \pi_{1}^{\prime \prime}$ is a free $\mathbf{Z}_{l}[[u, v]]$-module generated by $(x, y)$, where $(u+1) \cdot z=x \cdot z \cdot x^{-1}$ and $(v+1) \cdot z=y \cdot z \cdot y^{-1}$ for any $z \in \pi_{1}^{\prime} / \pi_{1}^{\prime \prime}$ (see [I1, Theorem 2]). It follows from (8.0.2) that $\sigma((x, y))=h_{\sigma}(u, v) \cdot(x, y)$, where $h_{\sigma}(u, v):=1+\sum_{i, j \geq 1} \alpha_{i, j}(\sigma) u^{i} v^{j}$. Coefficients $\beta_{i, j}: G_{\mathbf{Q}\left(\mu_{l} \infty\right)} \rightarrow \mathbf{Q}_{l}(i+j)$ are defined by the equality

$$
\log h_{\sigma}\left(e^{U}-1, e^{V}-1\right)=\sum_{i, j \geq 1} \frac{\beta_{i, j}(\sigma)}{i ! j !} U^{i} V^{j}
$$

(see [I1, pages 96 and 105]). We shall compare these coefficients with $l$-adic iterated integrals defined by us. 
The inclusion $k$ of $\pi_{1}\left(X_{\overline{\mathbf{Q}}}, \overrightarrow{01}\right)$ into $\mathbf{Q}_{l}\{\{X, Y\}\}$ given by $k(x)=e^{X}$ and $k(y)=e^{Y}$ induces an action of $\sigma$ on $\mathbf{Q}_{l}\{\{X, Y\}\}$ given by

$$
\sigma(X)=X \quad \text { and } \quad \sigma(Y)=\Lambda_{p}(\sigma)^{-1} \cdot Y \cdot \Lambda_{p}(\sigma)
$$

The logarithm of $\sigma, \log \sigma \in \operatorname{Der}^{*}\left(\mathbf{Q}_{l}\{\{X, Y\}\}\right)$ and

$$
(\log \sigma)(X)=0, \quad(\log \sigma)(Y)=[Y, \mathcal{L}(X, Y)(\sigma)]
$$

for some element $\mathcal{L}(X, Y)(\sigma) \in[L(X, Y), L(X, Y)]$. Let $L^{\prime}:=[L(X, Y)$, $L(X, Y)]$ and $L^{\prime \prime}:=\left[L^{\prime}, L^{\prime}\right]$. Then

$$
\mathcal{L}(X, Y)(\sigma)=\sum_{n=2}^{\infty} \sum_{i+j=n, i>0, j>0} a_{i j}(\sigma)\left[\cdots\left[\cdots[Y, X] X^{i-1}\right] Y^{j-1}\right] \bmod L^{\prime \prime}
$$

where $a_{i j}: G_{\mathbf{Q}\left(\mu_{l} \infty\right)} \rightarrow \mathbf{Q}_{l}(i+j)$. Hence

$$
(\log \sigma)(Y)=\sum_{n=2}^{\infty} \sum_{i+j=n, i>0, j>0} a_{i j}(\sigma)\left[\cdots\left[\cdots[X, Y] X^{i-1}\right] Y^{j}\right] \bmod L^{\prime \prime}
$$

We shall calculate the coefficients $a_{i, j}$.

LEMMA 8.0.5. We have $k\left(\left(y^{b-1}\left(x^{a-1}(x, y) \cdots\right) \cdots\right)=e^{r_{a, b}(X, Y)}\right.$, where

$$
\begin{aligned}
r_{a, b}(X, Y)=\sum_{i_{a}, \ldots, i_{1}, j_{b}, \ldots, j_{1} \geq 1} & \frac{(-1)^{i_{a}+\cdots+i_{1}+j_{b}+\cdots+j_{1}-1}}{i_{a} ! \cdots i_{1} ! \cdot j_{b} \cdots j_{1} !} \\
& \times\left[\cdots\left[\cdots[Y, X] X^{i_{a}+\cdots+i_{1}-1}\right] Y^{j_{b}+\cdots+j_{1}-1}\right] \bmod L^{\prime \prime} .
\end{aligned}
$$

Proof. First one calculates $r_{1,1}(X, Y)$ and next by induction $r_{a, b}(X, Y)$ for any pair $(a, b)$.

LEMMA 8.0.6. There is a continuous bijection of vector spaces

$$
L^{\prime} / L^{\prime \prime} \approx \mathbf{Q}_{l}[[s, t]]
$$

given by $\left[\cdots\left[\cdots[Y, X] X^{i-1}\right] Y^{j-1}\right] \rightarrow s^{i} t^{j}$. The element $r_{a, b}(X, Y) \in L^{\prime} / L^{\prime \prime}$ corresponds to a power series $-\left(e^{-s}-1\right)^{a}\left(e^{-t}-1\right)^{b}$. 
Observe that $\Lambda_{p}(\sigma)=e^{\varphi_{\sigma}(X, Y)}$, where $\varphi_{\sigma}(X, Y) \in L^{\prime}$. The action of $\sigma$ on $\mathbf{Q}_{l}\{\{X, Y\}\}$ induces

$$
\sigma: L(X, Y) / L^{\prime \prime} \longrightarrow L(X, Y) / L^{\prime \prime}
$$

given by $\sigma(X)=X$ and $\sigma(Y)=Y+\left[Y, \varphi_{\sigma}(X, Y)\right] \bmod L^{\prime \prime}$. It follows from (8.0.1) that

$$
\varphi_{\sigma}(X, Y)=\sum_{i, j \geq 1} \alpha_{i, j}(\sigma) r_{i, j}(X, Y) \bmod L^{\prime \prime} .
$$

We shall calculate $(\log \sigma)(Y)$, where $\sigma: L(X, Y) / L^{\prime \prime} \rightarrow L(X, Y) / L^{\prime \prime}$.

Proposition 8.0.8. The element $(\log \sigma)(Y) \in L^{\prime} / L^{\prime \prime}$ corresponds to the power series

$$
t \log \left(1+\sum_{i, j \geq 1} \alpha_{i, j}(\sigma)\left(e^{-s}-1\right)^{i}\left(e^{-t}-1\right)^{j}\right) \in \mathbf{Q}_{l}[[s, t]] .
$$

Proof. Let $F_{\sigma}(s, t) \in \mathbf{Q}_{l}[[s, t]]$ corresponds to $\varphi_{\sigma}(X, Y) \in L^{\prime} / L^{\prime \prime}$. Then the series $-t F_{\sigma}(s, t)$ corresponds to $(\sigma-I d)(Y)$, the series $t F_{\sigma}(s, t)^{2}$ corresponds to $(\sigma-I d)^{2}(Y)$, the series $t\left(-F_{\sigma}(s, t)\right)^{n}$ corresponds to $(\sigma-I d)^{n}(Y)$. Hence $(\log \sigma)(Y)$ corresponds to the series $t \log \left(1-F_{\sigma}(s, t)\right)$. It follows from Lemma 8.0.6 that $F_{\sigma}(s, t)=-\sum_{i, j \geq 1} \alpha_{i, j}(\sigma)\left(e^{-s}-1\right)^{i}\left(e^{-t}-1\right)^{j}$.

It follows from (8.0.4) and Proposition 8.0.8 that the coefficient $a_{i, j}(\sigma)$ is equal to the coefficient of the power series $-\log \left(1+\sum_{i, j \geq 1} \alpha_{i, j}(\sigma)\left(e^{-s}-\right.\right.$ $\left.1)^{i}\left(e^{-t}-1\right)^{j}\right)$ at $s^{i} t^{j}$. It follows from (8.0.3) that $\frac{\beta_{i, j}(\sigma)}{i ! j !}$ is the coefficient of the series $\log \left(1+\sum_{i, j \geq 1} \alpha_{i, j}(\sigma)\left(e^{U}-1\right)^{i}\left(e^{V}-1\right)^{j}\right)$ at $U^{i} V^{j}$. Hence we get that $\frac{\beta_{i, j}(\sigma)}{i ! j !}=(-1)^{i+j-1} a_{i, j}(\sigma)$. It follows from Proposition 5.1.8 that $(\log \sigma)(Y) \stackrel{=}{=}\left[Y,\left(\log \sigma_{p}\right)(1)\right]$. We recall that $\left(\log \sigma_{p}\right)(1)=\sum_{e \in \mathcal{B}} a_{p}^{e}(\sigma) e$, where $\mathcal{B}$ is a Hall base of $\operatorname{Lie}(X, Y)$. Hence we get that

$$
a_{i, j}(\sigma)=a_{p}^{\left[\cdots\left[\cdots[Y, X] X^{i-1}\right] Y^{j-1}\right]}(\sigma) .
$$

Therefore we have proved the following result.

Proposition 8.0.9. We have

$$
\frac{\beta_{i, j}(\sigma)}{i ! j !}=(-1)^{i+j-1} a_{p}^{\left[\left[[Y, X] X^{i-1}\right] Y^{j-1}\right]}(\sigma) .
$$




\section{REFERENCES}

[AI] G. W. Anderson and Y. Ihara, Pro-l branched coverings of $\mathbf{P}^{1}$ and higher circular l-units. Part 2, International Journal of Mathematics, Volume 1, No 2 (1990), 119-148.

[BD] A. A. Beilinson and P. Deligne, Interprétation motivique de la conjecture de Zagier reliant polylogarithmes et régulareurs, Motives (U. Jannsen, S. L. Kleiman and J.-P. Serre, eds.), Proc. of Symp. in Pure Math. 55, Part II, AMS (1994), pp. $97-121$.

[D] P. Deligne, Le groupe fondamental de la droite projective moins trois points, Galois Groups over Q (Y. Ihara, K. Ribet and J.-P. Serre, eds.), Mathematical Sciences Research Institute Publications, no 16 (1989), pp. 79-297.

[Dr] W. G. Drinfeld, On quasitriangular quasi-Hopf algebras and a group closely connected with $\operatorname{Gal}(\overline{\mathbf{Q}} / \mathbf{Q})$, Algebra i Analiz, 2 (1990), 114-148; English translation, Leningrad Math. J., 2 (4) (1991), 829-860.

[I1] Y. Ihara, Profinite braid groups, Galois representations and complex multiplications, Annals of Math., 123 (1986), 43-106.

[I2] Y. Ihara, Braids, Galois Groups and Some Arithmetic Functions,, Proc. of the Int. Cong. of Math. Kyoto (1990), 99-119.

[MKS] W. Magnus, A. Karrass, D. Solitar, Combinatorial Group Theory, Pure and Applied Mathematics, XIII, Interscience Publishers, 1966.

[N1] H. Nakamura, Galois rigidity of pure sphere braid groups and profinite calculus, J. Math. Sci. Univ. Tokyo, 1 (1994), 71-136.

[N2] H. Nakamura, Tangential base points and Eisenstein series, Aspects of Galois Theory (H. Volklein, D. Harbater, P. Muller and J. Thompson, eds.), London Math. Society Lecture Note Series 256 (1999), pp. 202-217.

[NT] H. Nakamura and H. Tsunogai, Some finiteness theorems on Galois centralizers in pro-l mapping class groups, Journal fur die reine und angewandte Mathematik, 441 (1993), 115-144.

[S1] Ch. Soulé, K-théorie des anneaux d'entiers de corps de nombres et cohomologie étale, Inventiones math., 55 (1979), 251-295.

[S2] Ch. Soulé, On higher p-adic regulators, Springer Lecture Notes N 854 (1981), 372-401.

[S3] Ch. Soulé, Eléments Cyclotomiques en K-Théorie, Asterisque, 147-148 (1987), 225-258.

[W1] Z. Wojtkowiak, The Basic Structure of Polylogarithmic Functional Equations, Structural Properties of Polylogarithms (L. Lewin, ed.), Mathematical Surveys and Monographs, Vol 37, pp. 205-231.

[W2] Z. Wojtkowiak, Monodromy of iterated integrals and non-abelian unipotent periods, Geometric Galois Actions London Math. Soc. L.N. Series 243, Cambridge University Press, pp. 219-289.

[W3] Z. Wojtkowiak, Mixed Hodge Structures and Iterated Integrals I, Motives, Polylogarithms and Hodge Theory. Part I: Motives and Polylogarithms, International Press Lecture Series Vol. 3, Part I (2002), pp. 121-208. 
[W4] Z. Wojtkowiak, On l-adic iterated integrals, Prépublication n 603, Université de Nice (2000).

Université de Nice-Sophia Antipolis

Département de Mathématiques

Laboratoire Jean Alexandre Dieudonné

U.R.A. au C.N.R.S., No 168

Parc Valrose - B.P.N $N^{\circ} 71$

06108 Nice Cedex 2

France

wojtkow@math.unice.fr 